\title{
Kinetostatic analysis and actuation strategy of a planar tensegrity 2-X manipulator
}

\author{
Matthieu Furet \\ Ecole centrale de Nantes, \\ Laboratoire des Sciences du Numérique de Nantes (LS2N) \\ UMR CNRS 6004, 1 rue de la Noë, 44321 Nantes, France \\ Email: matthieu.furet@Is2n.fr \\ Philippe Wenger \\ CNRS, \\ Laboratoire des Sciences du Numérique de Nantes (LS2N) \\ UMR CNRS 6004, 1 rue de la Noë, 44321 Nantes, France \\ Email: philippe.wenger@ls2n.fr
}

\begin{abstract}
A planar tensegrity manipulator made of two X-mechanisms in series is studied in this paper. Contrary to a classical 2-R linkage, the proposed architecture does not contain elements subject to bending and it can be driven with remote actuation and cables in an antagonistic way. Accordingly, it is an interesting candidate for the design of light-weight manipulators with variable stiffness suitable for safe interactions. On the other hand, its kinematics is more challenging because of a variable instantaneous center of rotation of the X-mechanisms. First, the inverse kinematic problem is solved using an adequate methodology, the singularities are determined and the workspace shape is analyzed as a function of the design parameters. Then two actuation schemes are studied and the wrench feasible workspace is analyzed for each of them. The second actuation scheme provides a larger wrench feasible workspace and allows for stiffness control.
\end{abstract}

\section{Introduction}

A tensegrity structure is an assembly of compressive elements (bars) and tensile elements (cables, springs) held together in equilibrium [1,2]. Tensegrity is known in architecture and art for more than a century [3] and is suitable for modeling living organisms [4]. Tensegrity mechanisms have been more recently studied for their promising properties in robotics such as low inertia, natural compliance and deployability [5-7]. They are also interesting candidates to design locomotion systems [8-11]. A tensegrity mechanism is obtained when one or several elements are actuated. This work falls withing the context of the AVINECK project involving biologists and roboticists with the main objective to model and design bird necks. Birds are capable of using their neck for dextrous tasks interacting with the environment (e.g. a vulture picking food from a carcass), as well as for tasks demanding high force transmissions and accelerations (e.g. the woodpecker hitting a tree trunk). Thus, bird necks are an interesting source of bio-inspiration for designing new manipulators with enhanced performances. Accordingly, a class of planar tensegrity manipulators made of a series assembly of several X-mechanisms i.e. crossed four-bar mechanisms with springs along their lateral sides, has been chosen as a suitable candidate for a preliminary planar model of a bird neck, see Fig. 1. These mechanisms are inspired from the Snelson's X-shape mechanisms [12]. Although simplified because it is planar, this model goes beyond the only available bird neck model in the literature that uses a simple planar articulated linkage [13], as it can be more easily actuated with cables that play the role of tendons and muscles. Moreover, the center of rotation of the X-mechanism is not fixed, which is the case in most biological joints. Snelson's $\mathrm{X}$-shape mechanisms have been studied by a number of researchers, either as single mechanisms $[5,7,14,15]$ or assembled in series [11,16-21]. In this paper, the manipulator may be subject to gravity unlike in [11], where the mechanism was used in a snake-like manipulator moving on the ground. This paper is twofold. In the first part, the full kinematic analysis of the manipulator is performed. Since each X-mechanism has a variable instantaneous center of rotation, the symbolic calculation of the inverse geometric problem is more challenging: polynomials of excessive degree may appear if the problem is not 


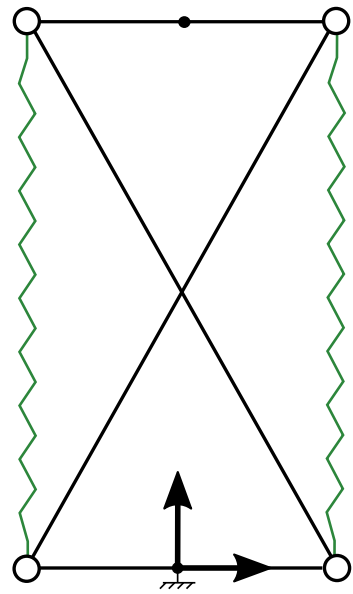

(a)

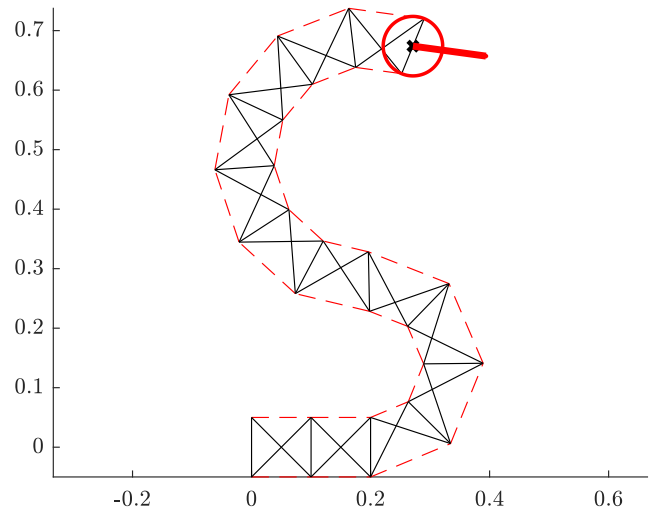

(b)

Fig. 1: An X-mechanism with two lateral springs $(a)$ and a series assembly mimicking a bird neck $(b)$

tackled with care. A practical method is provided that makes it possible to come up with a quartic polynomial, which turns out to be of minimal degree. The singularities of the manipulator are shown to divide the joint space into only two singularity-free components or aspects. Depending on the values of its geometric parameters, the manipulator at hand may be cuspidal, i.e. it can move from one inverse kinematic solution to another without meeting a singularity. A necessary and sufficient condition is stated for the manipulator to be cuspidal. The effect of joint limits is analyzed and the accessibility regions are further classified according to the admissible configurations of each X-mechanism in these regions. The second part of this paper is dedicated to the static analysis and the determination of the wrench feasible workspace for two different actuation strategies. In the first actuation strategy, the manipulator is actuated with only two symmetric cables. With this actuation strategy, it is shown that the wrench feasible workspace is limited and stiffness cannot be controlled. The second actuation strategy proposed is an over-actuated antagonist strategy that produces a much larger wrench feasible workspace. Moreover, this actuation strategy allows tuning the manipulator stiffness, which can be of interest for safe interaction and collaborative robotics.

\section{Kinematic analysis}

\subsection{Manipulator description and parametrization}

The manipulator consists of a series assembly of two X-mechanisms as shown in Fig. 2. Unlike the original Snelson's $\mathrm{X}$-shape tensegrity that has only two compressive elements (the two crossing bars) and four tensile elements (the four sides), the X-mechanisms used in this paper have four bars in total. The two additional bars replace the tensile elements on the base and top sides. Accordingly, the X-mechanisms used in this study are class-3 tensegrities [3]. Each X-mechanism $i$ has a base bar and a top bar of length $b$ and two crossed bars of length $L$, thus defining an antiparallelogram. The mechanism assembly condition is $b \leq L$ and the particular case $b=L$ defines a degenerate manipulator as shown further. The springs have no impact on the kinematic analysis and have been hidden in Fig. 2 for more simplicity. We need to define a suitable joint variable that describes each mechanism configuration without any ambiguity. In [11], the mechanism configuration was chosen as the orientation $\alpha_{i}$ of the upper bar with respect to the base bar, but such a choice is allowed for mechanism motions restricted to $-\pi<\alpha_{i}<\pi$. For a matter of completeness in the kinematic analysis, we are interested in allowing each mechanism to move within its full range beyond its two singularities where it assumes a flat configuration. Since the upper bar makes two turns for one mechanism full turn, $\alpha_{i}$ is not appropriate to define the mechanism configuration. Let us introduce a line segment of length $l_{i}$ that links the middle points of the top and base bars of each mechanism $i$ (shown in dotted line in Fig. 2). The angle between this line and the direction orthogonal to the base bar is referred to as $\theta_{i}$. For the symmetric design considered here, it can be shown that $\alpha_{i}=2 \theta_{i}$. Thus, when the mechanism makes a full turn: $-\pi<\theta_{i}<\pi$. Assuming that each mechanism remains always in the crossed-bar assembly mode, the manipulator configuration can thus be unambiguously defined with $\left(\theta_{1}, \theta_{2}\right)$. Besides, additional angles, namely $\phi_{i}$ and $\psi_{i}$ are considered as they will be useful to derive the inverse kinematics. Since the mechanism sides define an isosceles trapezoid, the length $l_{i}$ of the line segment that links the middle points of the top and base bars can be expressed as follows:

$$
l_{i}\left(\theta_{i}\right)=\sqrt{L^{2}-b^{2} \cos ^{2}\left(\theta_{i}\right)}
$$




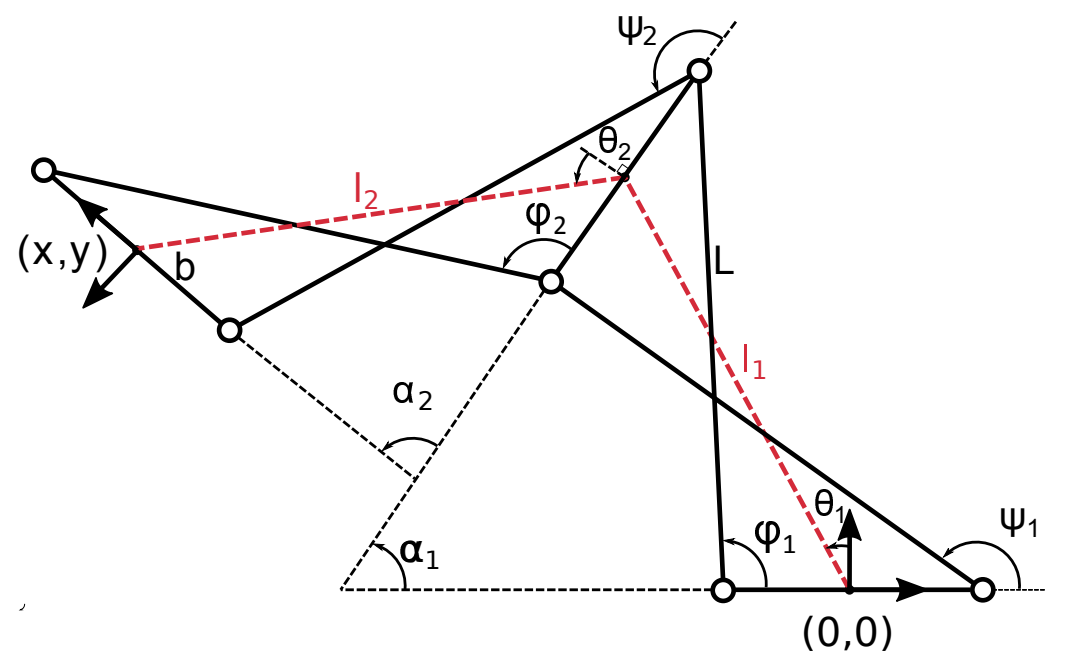

Fig. 2: Manipulator description

\subsection{Direct Kinematics}

To establish the direct kinematic equations, we start with the base frame centered at the middle point of the base bar with the $\mathrm{x}$-axis aligned along this bar. The reference point $(x, y)$ is chosen as the middle point of the top-bar (Fig. 2). The direct kinematic equations of a manipulator with $n$ mechanisms in series can be written as a virtual serial $n \mathrm{R}$ manipulator with (varying) link lengths $l_{i}$ and joint angles $\theta_{i}$ :

$$
\left\{\begin{array}{l}
x=-\sum_{i=1}^{n} l_{i}\left(\theta_{i}\right) \sin \left(-\theta_{i}+\sum_{j=1}^{i} 2 \theta_{j}\right) \\
y=\sum_{i=1}^{n} l_{i}\left(\theta_{i}\right) \cos \left(-\theta_{i}+\sum_{j=1}^{i} 2 \theta_{j}\right)
\end{array}\right.
$$

For a manipulator made of two mechanisms in series with input variables $\theta_{1}$ and $\theta_{2}$, the direct kinematic equations simplifies to:

$$
\left\{\begin{array}{l}
x=-l_{1}\left(\theta_{1}\right) \sin \left(\theta_{1}\right)-l_{2}\left(\theta_{2}\right) \sin \left(2 \theta_{1}+\theta_{2}\right) \\
y=l_{1}\left(\theta_{1}\right) \cos \left(\theta_{1}\right)+l_{2}\left(\theta_{2}\right) \cos \left(2 \theta_{1}+\theta_{2}\right)
\end{array}\right.
$$

where $l_{1}$ and $l_{2}$ were defined in Eq. (1).

\subsection{Inverse Kinematics}

Deriving the so-called characteristic polynomial establishes a univariate polynomial to solve the inverse kinematics. The direct kinematic equations (3) have been derived in their most compact form, namely, the two output variables $x$ and $y$ are expressed as functions of the two input variables $\theta_{1}$ and $\theta_{2}$ like in a $2 \mathrm{R}$ serial manipulator. However, starting from these equations to derive a characteristic polynomial is not appropriate. Indeed, the square roots appearing in $l_{1}$ and $l_{2}$ would have to be first cleared out, with the consequence of raising the degree of the resulting characteristic polynomial and providing spurious solutions. Instead, it is better to write $x$ and $y$ as functions of the crossed-bar angles $\phi_{1}$ and $\psi_{2}$ as follows:

$$
\left\{\begin{array}{l}
x=-\frac{b}{2}+L \cos \left(\phi_{1}\right)+L \cos \left(\psi_{2}+\alpha_{1}\right)+\frac{1}{2} b \cos \left(\alpha_{1}+\alpha_{2}\right) \\
y=L \sin \left(\phi_{1}\right)+L \sin \left(\psi_{2}+\alpha_{1}\right)+\frac{1}{2} b \sin \left(\alpha_{1}+\alpha_{2}\right)
\end{array}\right.
$$

In addition, by deriving the anti-parallelogram loop-closure for mechanism $i$ projected on $\mathrm{x}$ and $\mathrm{y}$ axis, we have:

$$
\left\{\begin{array}{l}
b+L \cos \left(\psi_{i}\right)+b \cos \left(\alpha_{i}\right)-L \cos \left(\phi_{i}\right)=0 \\
L \sin \left(\psi_{i}\right)+b \sin \left(\alpha_{i}\right)-L \sin \left(\phi_{i}\right)=0
\end{array}\right.
$$


By rearranging Eqs. (5) for each mechanism, $\psi_{1}$ and $\phi_{2}$ can be eliminated. Finally, the following two loop-closure equations below are obtained:

$$
\left\{\begin{array}{l}
-2 L b \sin \left(\alpha_{1}\right) \sin \left(\phi_{1}\right)-2 L b\left(\cos \left(\alpha_{1}\right)+1\right) \cos \left(\phi_{1}\right)+2 b^{2}\left(\cos \left(\alpha_{1}\right)+1\right)=0 \\
2 L b \sin \left(\alpha_{2}\right) \sin \left(\psi_{2}\right)+2 L b\left(\cos \left(\alpha_{2}\right)+1\right) \cos \left(\psi_{2}\right)+2 b^{2}\left(\cos \left(\alpha_{2}\right)+1\right)=0
\end{array}\right.
$$

A set of four equations in four unknowns is thus available. A univariate polynomial can be obtained upon elimination of three of the four unknowns. There are several ways to do so with a computer algebra software. We have used Maple and its Siropa library [22]. This library was developed in the frame of a collaborative project on the kinematics of parallel manipulators, see e.g. [23, 24] for more details on the implementation and use of these tools. It contains specific macrofunctions that use efficient algebraic tools such as Groebner bases. Specifically, the Projection function was used to project the system of four equations in order to obtain one single equation in one single variable, chosen here as $\phi_{1}$. The half-tangent substitution yields a factored polynomial and one of the factors defines the characteristic polynomial. The characteristic polynomial obtained turns out to be of degree 4 in $t=\tan \left(\phi_{1} / 2\right)$ and can be written as follows:

$$
a_{4} t^{4}+a_{3} t^{3}+a_{2} t^{2}+a_{1} t+a_{0}=0
$$

where :

$$
\begin{gathered}
a_{4}=(b+1)^{2}\left(b^{2} y^{2}+x^{4}+2 x^{2} y^{2}+y^{4}+4 x^{3}+4 x y^{2}+5 x^{2}+y^{2}+2 x\right) \\
a_{3}=4 y(b+1)\left(2 b^{2} x+b^{2}-2 x^{2}-2 y^{2}-4 x-1\right) \\
a_{2}=2\left(b^{4} y^{2}+b^{2} x^{4}+2 b^{2} x^{2} y^{2}+b^{2} y^{4}+b^{2} x^{2}-10 b^{2} y^{2}+x^{4}+2 x^{2} y^{2}+y^{4}-3 x^{2}+9 y^{2}\right) \\
a_{1}=4 y(b-1)\left(2 b^{2} x-b^{2}+2 x^{2}+2 y^{2}-4 x+1\right) \\
a_{0}=(b-1)^{2}\left(b^{2} y^{2}+x^{4}+2 x^{2} y^{2}+y^{4}-4 x^{3}-4 x y^{2}+5 x^{2}+y^{2}-2 x\right)
\end{gathered}
$$

Note that $L$ was set equal to 1 for to simplify the calculations without loss of generality. For each solution $\phi_{1}$, $\alpha_{1}$ is then solved from the first equation in (6). Disregarding the solution $\alpha_{1}=\pi$ corresponding to the parallelogram assembly, one obtains:

$$
\tan \left(\alpha_{1} / 2\right)=\frac{-\cos \left(\phi_{1}\right)+b}{\sin \left(\phi_{1}\right)}
$$

Finally, $\psi_{2}$ and $\alpha_{2}$ are solved from system (4), which gives two solutions, one of which never satisfies the closed-loop equations (6). In total, thus, one comes up with a maximum number of four solutions. The inverse kinematics is solved for a manipulator defined by $L=1$ and $b=2 / 3$ at $x=0.03$ and $y=1.6$. Four solutions are found (see Fig. 3), showing that the characteristic polynomial (7) is of minimal degree. 

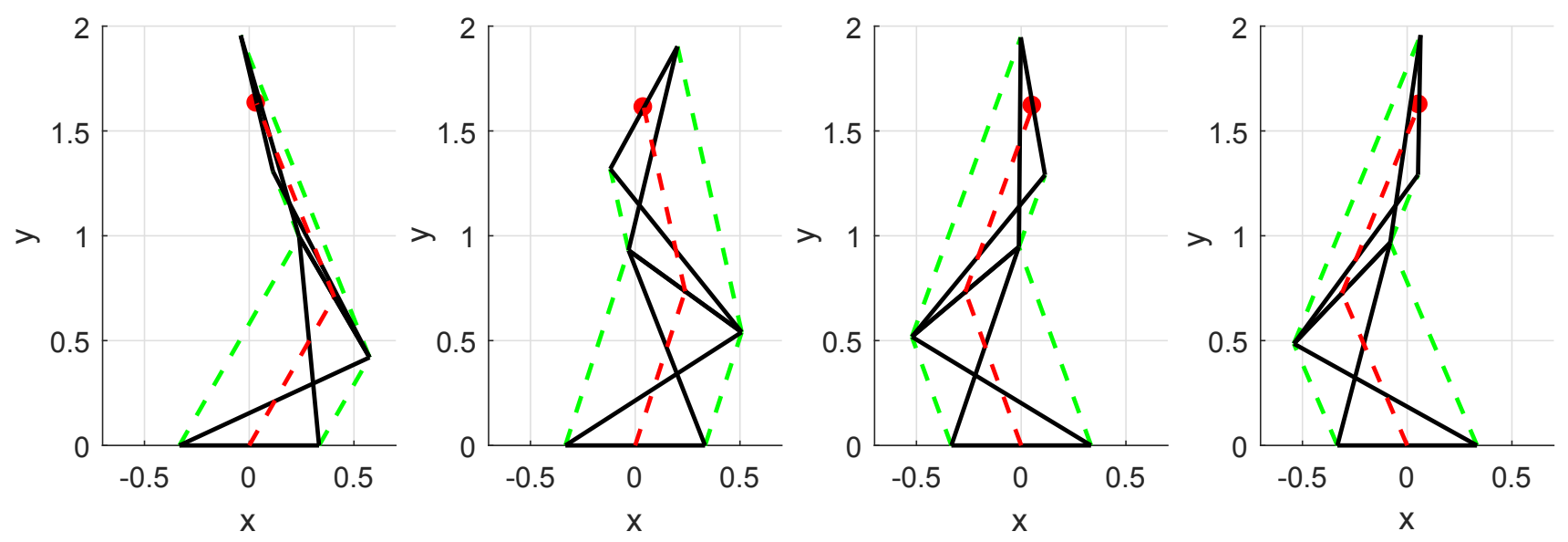

Fig. 3: The four inverse solutions at $x=0.03$ and $y=1.6(L=1$ and $b=2 / 3)$

\subsection{Singularity analysis and aspects}

The Jacobian matrix $\mathbf{J}$ of the manipulator, defined such that $[\dot{x}, \dot{y}]^{\top}=\mathbf{J}\left[\dot{\theta_{1}}, \dot{\theta_{2}}\right]^{\top}$, is derived from system (3):

$$
\mathbf{J}=\left(\begin{array}{ll}
\frac{\partial x}{\partial \theta_{1}} & \frac{\partial x}{\partial \theta_{2}} \\
\frac{\partial y}{\partial \theta_{1}} & \frac{\partial y}{\partial \theta_{2}}
\end{array}\right)
$$

with :

$$
\left\{\begin{array}{l}
\frac{\partial x}{\partial \theta_{1}}=-\frac{-2 b^{2} \cos \left(\theta_{1}\right)^{3}+L^{2} \cos \left(\theta_{1}\right)+b^{2} \cos \left(\theta_{1}\right)+2 \sqrt{L^{2}-b^{2} \cos \left(\theta_{2}\right)^{2}} \cos \left(2 \theta_{1}+\theta 2\right) \sqrt{L^{2}-b^{2} \cos \left(\theta_{1}\right)^{2}}}{\sqrt{L^{2}-b^{2} \cos \left(\theta_{1}\right)^{2}}} \\
\frac{\partial x}{\partial \theta_{2}}=-\frac{-\sin \left(2 \theta_{1}+\theta_{2}\right) b^{2} \cos \left(\theta_{2}\right) \sin \left(\theta_{2}\right)-\cos \left(2 \theta_{1}+\theta_{2}\right) \cos \left(\theta_{2}\right)^{2} b^{2}+L^{2} \cos \left(2 \theta_{1}+\theta_{2}\right)}{\sqrt{L^{2}-b^{2} \cos \left(\theta_{2}\right)^{2}}} \\
\frac{\partial y}{\partial \theta_{1}}=-\frac{-2 b^{2} \cos \left(\theta_{1}\right)^{2} \sin \left(\theta_{1}\right)+L^{2} \sin \left(\theta_{1}\right)+2 \sqrt{L^{2}-b^{2} \cos \left(\theta_{2}\right)^{2}} \sin \left(2 \theta_{1}+\theta 2\right) \sqrt{L^{2}-b^{2} \cos \left(\theta_{1}\right)^{2}}}{\sqrt{L^{2} b^{2} \cos \left(\theta_{1}\right)^{2}}} \\
\frac{\partial y}{\partial \theta_{2}}=-\frac{-\cos \left(2 \theta_{1}+\theta_{2}\right) b^{2} \cos \left(\theta_{2}\right) \sin \left(\theta_{2}\right)-\sin \left(2 \theta_{1}+\theta_{2}\right) \cos \left(\theta_{2}\right)^{2} b^{2}+L^{2} \sin \left(2 \theta_{1}+\theta_{2}\right)}{\sqrt{L^{2}-b^{2} \cos \left(\theta_{2}\right)^{2}}}
\end{array}\right.
$$

Its determinant is derived and, after clearing the numerator $\sqrt{L^{2}-b^{2} \cos \left(\theta_{1}\right)^{2}} \sqrt{L^{2}-b^{2} \cos \left(\theta_{2}\right)^{2}}$ that never vanishes since $b<L$, the following expression is obtained:

$$
\begin{aligned}
\operatorname{Det}(\mathbf{J})= & \left(L^{2}-b^{2}\right) \cos (\theta 1) \sin (\theta 2)\left(L^{2}-2 b^{2} \cos (\theta 2)^{2}\right)+\sin (\theta 1) \cos (\theta 2)\left(L^{2}\left(L^{2}+b^{2}\right)-2 L^{2} b^{2} \cos (\theta 2)^{2}\right) \\
& -2 \sin (\theta 2) \cos (\theta 2) \sqrt{-b^{2} \cos (\theta 1)^{2}+L^{2}} \sqrt{L^{2}-b^{2} \cos (\theta 2)^{2}} b^{2}
\end{aligned}
$$

The solutions to $\operatorname{Det}(\mathbf{J})=0$ should account for the singularity conditions occuring when the instantaneous centers of rotation (ICR) of the two X-mechanisms are aligned with the end-effector control point. A particular situation arises when the two X-mechanisms are coincident. This situation is equivalent to the fully-folded back configuration of a planar $2 \mathrm{R}$ serial manipulator with equal link lengths. When the manipulator is in this singularity, the reference point remains at the origin $x=0$ and $y=0$. This particular singularity is defined by $\theta_{1}=-\theta_{2} \pm \pi$ and it can be verified that $\operatorname{Det}(\mathbf{J})$ vanishes in these configurations. The general singularity is like the fully outstretched configuration of a planar $2 \mathrm{R}$ serial manipulator. In this singularity, the manipulator cannot produce an instantaneous motion along a line passing through the two ICR and the endeffector control point. Unexpectedly, it was possible to derive an explicit form of this general solution. When $\cos \left(\theta_{2}\right) \neq 0$, this solution can be written as:

$$
\tan \left(\theta_{1}\right)=\frac{-\sin \left(\theta_{2}\right)\left(\left(2 b^{2} \cos \left(\theta_{2}\right)^{2}-L^{2}\right)^{2}-L^{2} b^{2}\right)}{\cos \left(\theta_{2}\right)\left(\left(2 b^{2} \cos \left(\theta_{2}\right)^{2}-L^{2}\right)^{2}+3 L^{2} b^{2}-4 \cos \left(\theta_{2}\right)^{2} b^{4}\right)}
$$




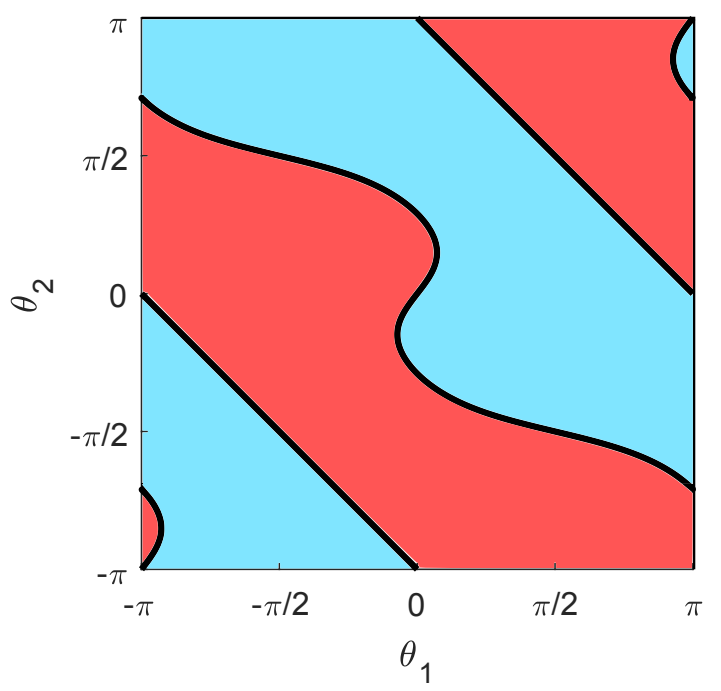

Fig. 4: Singularity curves in the joint space and aspects when $L=1$ and $b=2 / 3$

When $\cos \left(\theta_{2}\right)=0$, then $\cos \left(\theta_{1}\right)=0$, which means that both mechanisms are in their flat singularity. Finally, it can be shown that the second factor of the denominator in (13) never vanishes.

The above singularities divide the joint space into singularity-free domains called aspects [25]. The singularity $\theta_{1}=$ $-\theta_{2} \pm \pi$ produces two lines while the singularity defined by Eq. (13) gives a curve, as shown in Fig. 4. In the absence of joint limits, the opposite sides of the square are in fact coincident and the singularity curves divide the joint space into only two aspects. Since the manipulator admits up to four solutions, there are necessarily more than one solution in one of the aspects. In fact, there are two solutions in each aspect, which can be verified by calculating the inverse solutions at any point in a four-solution region. This means that the manipulator can move from one inverse kinematic solution to another without meeting a singularity, namely, it is cuspidal $[26,27]$.

\subsection{Workspace analysis}

When mapped to Cartesian space, the singularity curves define the external and internal boundaries of the workspace. The equation of these curves can be obtained by deriving the discriminant of the characteristic polynomial (7). By doing so, a polynomial of degree 16 in $x$ and $y$ is obtained. Its expression is quite large and shall not be reported here but can be found in [28]. Figure 5 shows the plot of these singularity curves for three examples. In the middle and right figures, they divide the workspace into three regions. The number of solutions in each regions can be established by counting the number of solutions at any point in each region. In the largest one, the manipulator admits two inverse kinematic solutions. In the two smaller regions, there are four solutions. In the left figure, no internal boundaries exist and the manipulator admits two inverse solutions throughout its workspace. There are two degenerate designs, namely, $b=L$ and $b=0$. When $b=L$, each mechanism degenerates to a bar (the top and base bars coincide and so do their left (resp. right) joints). Thus, these two bars can move with a pure rotation about any of their two revolute joints. Accordingly, the manipulator behaves like two $2 \mathrm{R}$ planar linkages of link lengths $b$ and $b / 2$, respectively, with their base joints located at $x=-b / 2$ and $x=b / 2$, respectively (the reason for having $b / 2$ is that the base frame and the end-effector frame are located in the middle of the base and top bars, respectively). Thus, the workspace is the union of two hollow disks of outer (resp. inner) radius $3 b / 2$ (resp. $b / 2$ ). There are four solutions in the overlapping regions of the non-empty areas of the two hollow disks and two solutions anywhere else (see Fig. 6, left). When $b=0$, the manipulator degenerates to a planar 2R linkage and its workspace is a full disk of radius $2 L$, with two inverse kinematic solutions everywhere, see Fig. 6, right. These two degenerate situations were mentioned for the sake of completeness in the kinematic analysis but they have no practical interest and should be avoided.

The workspace boundaries are associated with the singularities of the 2-X manipulator as a whole. On the other hand, each X-mechanism has its own singularities occurring when it is completely flat. These singularities are defined by $\theta_{i}= \pm \frac{\pi}{2}$. The role of the $\mathrm{X}$-mechanism singularities is to distinguish mechanism solutions in right side up configurations (the top bar is above the base bar) from those in upside down configurations (the top bar is below the base bar). These two configurations are referred to as configuration up and configuration down, respectively, and illustrated in Fig. 7.

The curves associated with the singularities of the X-mechanisms are now plotted in the workspace, see Fig. 8. The additional curves divide the 2 -solution and the 4 -solution regions into smaller regions associated with different combinations of mechanism configurations as explained in Fig. 8. For example, the colored region with both $\|$ and // lines means that 


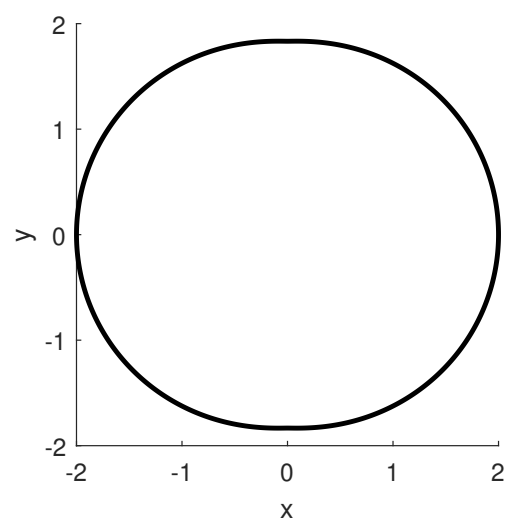

(a)

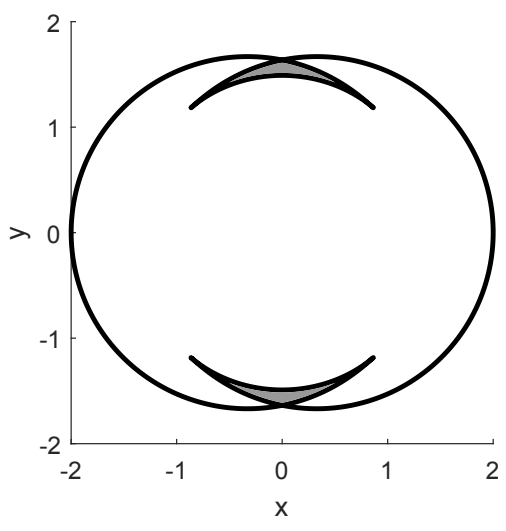

(b)

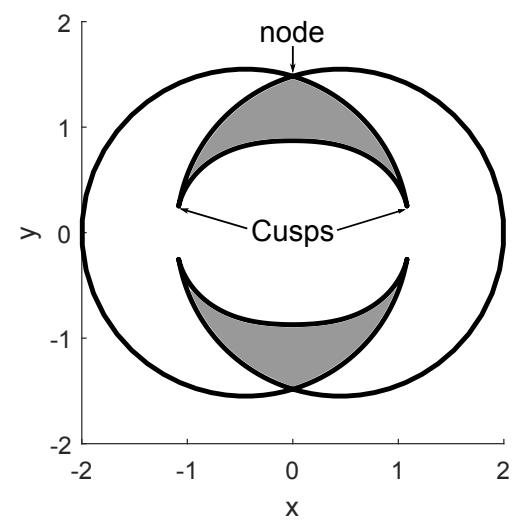

(c)

Fig. 5: Workspace boundaries when $L=1$ and $b=2 / 5(a), b=2 / 3(b), b=9 / 10(c)$. Four-solutions regions are filled in gray

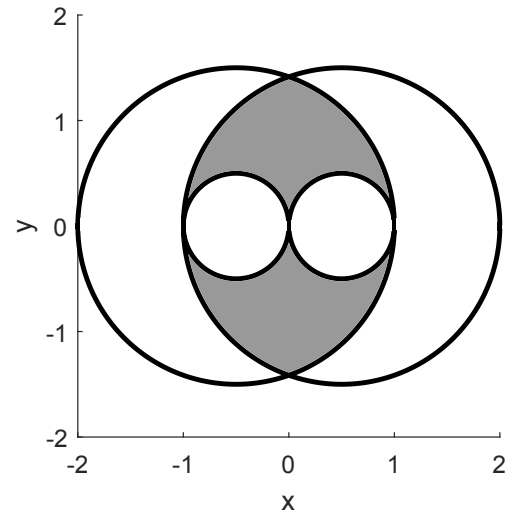

(a)

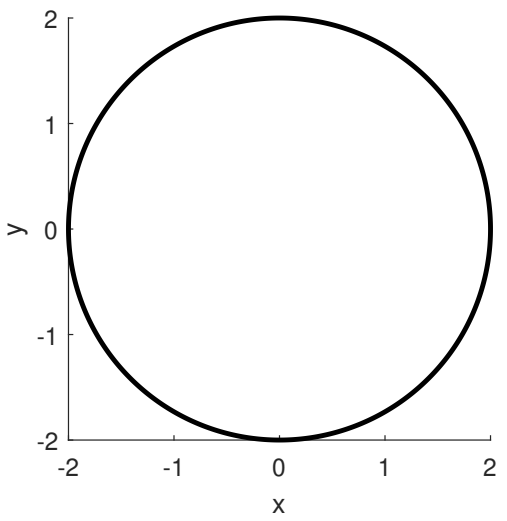

(b)

Fig. 6: Workspace boundaries for the two degenerate designs $b=L=1(a)$ and $b=0, L=1(b)$. Four-solutions regions are filled in gray

two solutions are of the type $M_{1} M_{2}$ (both mechanisms are up) and the other two are $M_{1} \overline{M_{2}}$ (the first mechanism is up and the second is down). In the context of a tensegrity manipulator driven with cables along the sides of the mechanisms, the reachable workspace is reduced to those regions where at least one solution exists with both mechanisms up. Those regions have vertical lines in Fig. 8. This point will be discussed in section 3.

\subsection{Cuspidality condition}

As shown above, the workspace may have four-solutions regions or not depending on the values of its geometric parameters. When two 4-solution regions exist, their boundaries have three singular points: a node and two cusps as shown in Fig. 5 , right. The existence of cusps shows that the manipulator is cuspidal as a non-singular solution changing motion can be defined by encircling one of the cusps [26,27].

From Fig. 5, the 4-solution regions are smaller when $b$ is smaller. In fact, the four-solution regions may even vanish (Fig. 5, left) and so do also the cusp points. The transition, called swallowtail bifurcation [29], occurs when the two cusps and the node coincide and define a point with four coincident inverse kinematic solutions. Thus, a geometric condition for having two cusps and four inverse solutions can be established by deriving a condition on the geometric parameters for the 4th-order characteristic polynomial to admit a quadruple root. The latter condition can be stated as follows [30]: 


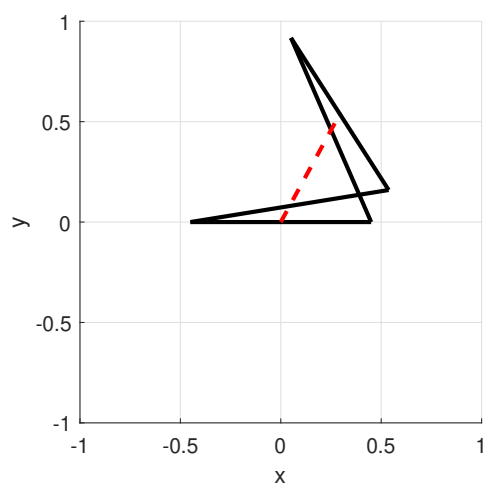

(a)

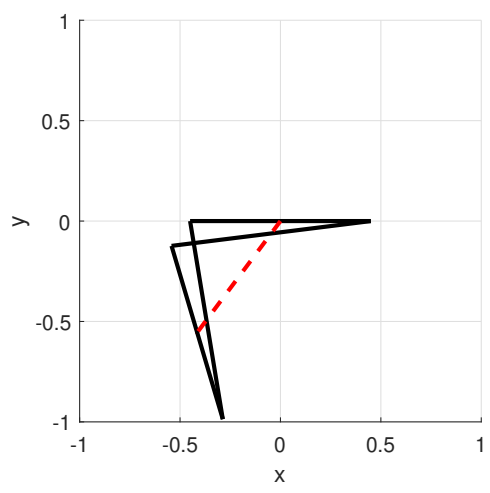

(b)

Fig. 7: X-mechanism in configuration up (a) and down (b)

$$
\left\{\begin{array}{l}
a_{4} a_{0}-\frac{1}{4} a_{3} a_{1}+\frac{1}{12} a_{2}^{2}=0 \\
\frac{1}{6} a_{4} a_{2} a_{0}-\frac{1}{16} a_{4} a_{1}^{2}-\frac{1}{16} a_{3}^{2} a_{0}+\frac{1}{48} a_{3} a_{2} a_{1}-\frac{1}{216} a_{2}^{3}=0 \\
\frac{1}{6} a_{4} a_{2}-\frac{1}{16} a_{3}^{2}=0
\end{array}\right.
$$

The above system can be simplified by observing that the quadruple root should appear at $x=0$ due to symmetry. Solving $b$ from the last two equations in Eq. (14) yields $b=\sqrt{6} / 6$. When $b>\sqrt{6} / 6$, the manipulator is cuspidal and has four inverse solutions, otherwise it has only two solutions and it is non-cuspidal.

This cuspidality condition was derived based on the assumption that cusps could appear only from a swallowtail bifurcation. While other transitions are possible in general (lips and beaks [29]), this assumption was set on the basis of intensive observations of the singularity plots for varying values of $b$. For the sake of rigor, the cuspidality condition was double checked by deriving the general existence condition of cusps. The solution set of cusps in $x$ and $y$ was obtained by eliminating $t$ from the characteristic polynomial and its first and second derivatives [27]. The discriminant of the resulting system was then derived, providing a factored expression in $b$ with 6 factors. Only one factor was found to have real roots, namely $6 b^{2}-1$, which matches the previous condition and confirms that the swallowtail is the only possible bifurcation of the singularity locus.

Keeping in mind that $L$ was set to 1 and taking into account the assembly condition, the cuspidality condition is actually $b<L<b \sqrt{6}$. The following result can thus be stated as follows:

a planar 2-degree-of-freedom manipulator made of two identical symmetrical X-mechanisms with crossed link lengths $L$ and base link $b$ is cuspidal and has four inverse kinematic solutions if and only if $b<L<b \sqrt{6}$.

To know whether a manipulator is cuspidal or not is very useful for trajectory planning and control [27].

\subsection{Influence of the joint limits}

Unlimited joints were assumed thus far, but for a practical implementation, joint limits will have to be considered. To determine the workspace boundaries in the presence of joint limits, one has to plot the lines associated with these joint limits into the Cartesian space, in addition to the singularity curves. Figure 9 shows how the workspaces with unlimited joints depicted in Fig. 5 change when $-\pi / 2<\theta_{1}<\pi / 2$ and $-\pi / 2<\theta_{2}<\pi / 2$. As compared with Fig. 8, the workspace of the middle figure in Fig. 9 is now reduced to the regions with vertical lines, namely, where both mechanisms are in their right side up configuration. This is because the joint limits restrict the mechanisms from turning upside-down. Note that this restriction is necessary in a remote actuation scheme with active cables since this scheme does not allow passing the flat singular configuration. The number of solutions is determined in each region by solving the inverse kinematics for an arbitrary point. As shown in Fig. 9, the main part of the workspace has only one solution. When $b=2 / 3$ (middle), the 4 -solution region is limited to the small upper diamond region (the one with only vertical lines in the zoomed-in view of Fig. 8). 


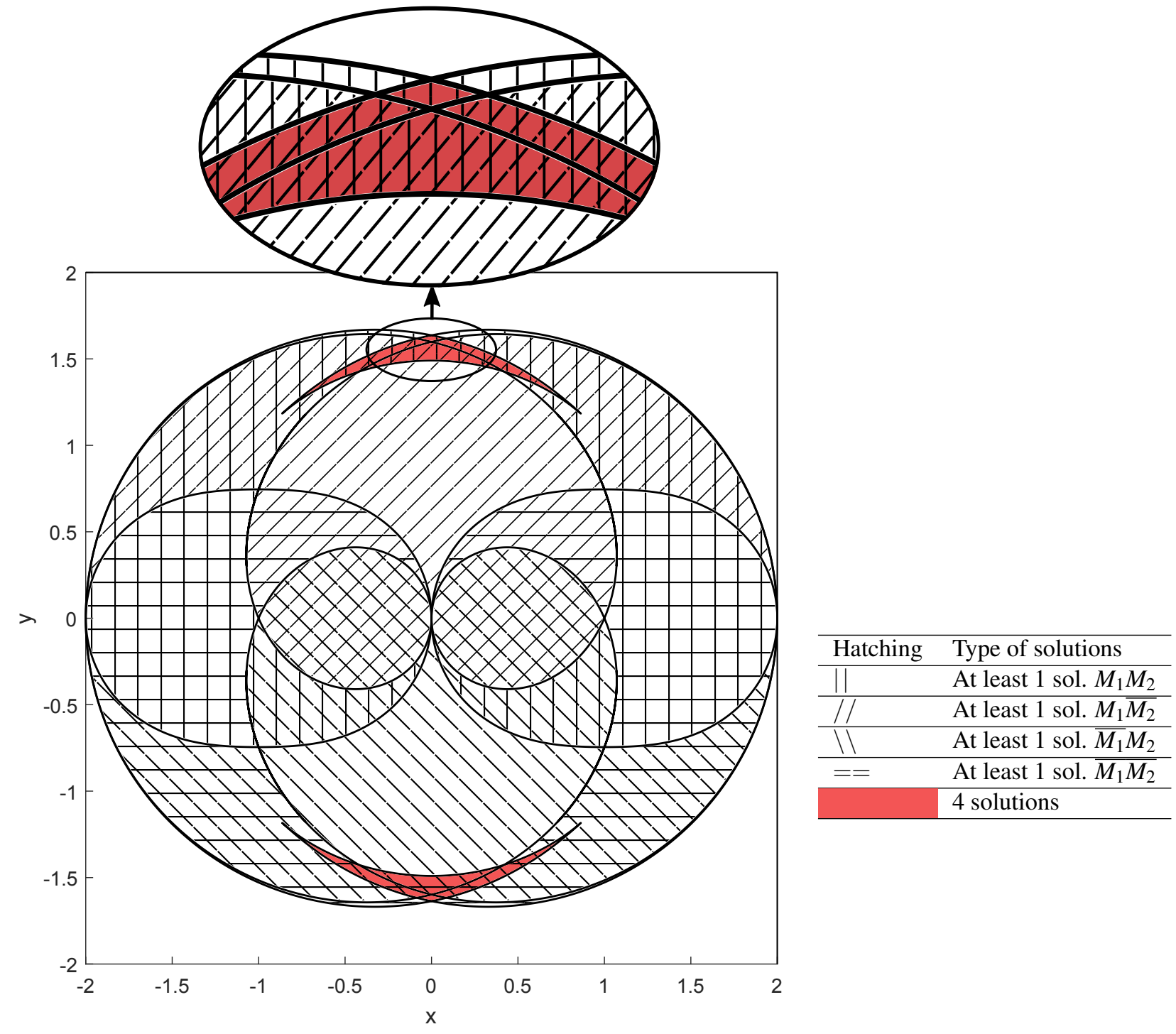

Fig. 8: workspace regions with different numbers and types of solutions $\left(L=1, b=2 / 3 . \overline{M_{i}}\right.$ (resp. $\left.M_{i}\right)$ means that mechanism $i$ is in configuration down (resp. up)

\section{Actuation and static analysis}

\subsection{Actuation strategy}

We now focus on the actuation of the tensegrity manipulator at hand. Several actuation schemes are possible, including embedded and remote actuation. Here a remote actuation scheme with active cables is considered. This actuation scheme allows for a light weight design since the actuators are removed from the moving elements. Then, it is necessary to define an approriate number of motors and a suitable way of routing the cables from the motors to the mechanisms. Regarding the number of motors, the minimum number is two since this particular planar tensegrity manipulator has two degrees of freedom. On the other hand, a redundant actuation scheme with four motors would allow each X-mechanism to be antagonistically actuated by active cables along its two sides. With such an antagonistic actuation scheme, we already know that the stiffness of a single mechanism can be tuned and a larger wrench feasible workspace can be obtained [15]. Regarding the routing strategy of the cables, several schemes can be considered, including strut-routed [16,31] and side-routed [11,19]. Two actuation strategies are studied in this section, one with two actuators and another with four actuators. Since the manipulator is remotely actuated with active cables, each mechanism must be constrained to operate in its up configuration. Accordingly, the rotations range of each mechanism is now restricted to $-\frac{\pi}{2}<\theta_{i}<\frac{\pi}{2}$.

\subsection{Static and stiffness modeling}

Several hypotheses are considered: (i) the cables are assumed mass-less and infinitely stiff, (ii) all the joints are assumed friction-less and with no clearances, (iii) the cable are assumed to move freely through the nodes without friction and, (iv) all 

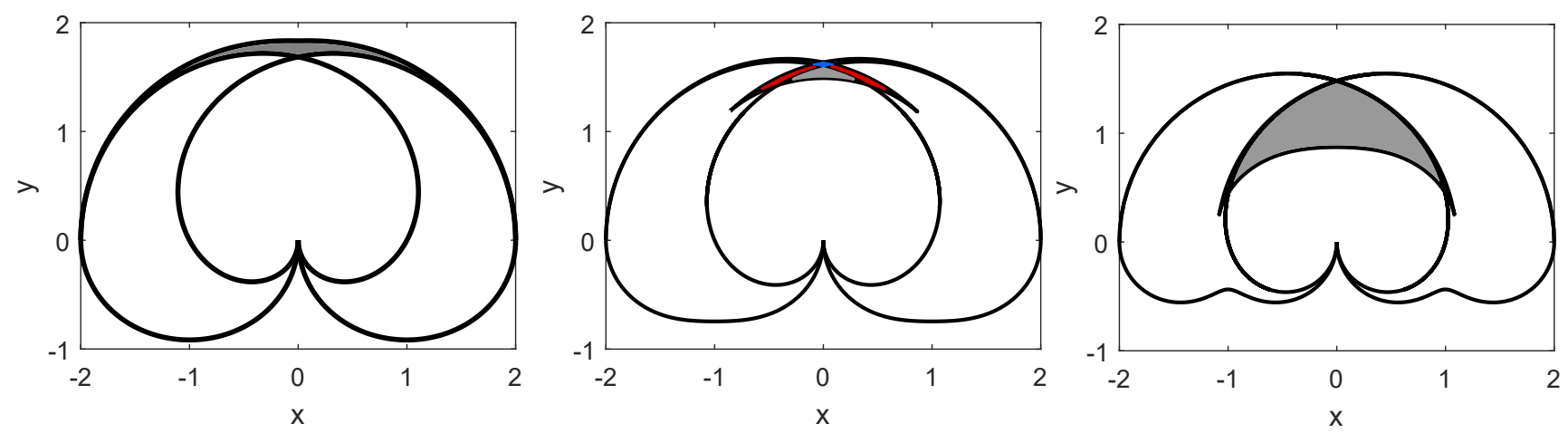

Fig. 9: workspace boundaries when $-\pi / 2<\theta_{1}, \theta_{2}<\pi / 2$, and for $L=1, b=2 / 5$ (left), $b=2 / 3$ (center), $b=9 / 10$ (right). the nonempty part is depicted with vertical lines. The number of solutions is defined by the filling color: none $=1$, grey $=2$, red $=3$ and blue $=4$

springs are linear tension springs. The static equations relate the input forces and the manipulator configuration. To obtain them, the static equilibrium condition must be established. A convenient way is to derive the total potential energy $U_{\text {total }}$ of the tensegrity system at hand. In the absence of external forces and assuming that the cables and the bars are infinitely stiff, $U_{\text {total }}$ is composed of the potential energy associated with the springs and the gravity, plus the work produced by the cables, denoted $U_{\text {springs }}, U_{\text {gravity }}$ and $U_{\text {cables }}$, respectively:

$$
U_{\text {total }}=U_{\text {cables }}+U_{\text {springs }}+U_{\text {gravity }}
$$

Let $\left(f_{r i}, f_{l i}\right),\left(l_{r i}, l_{l i}\right),\left(k_{r i}, k_{l i}\right)$ and $\left(l_{r 0 i}, l_{l 0 i}\right)$ refer to the cable forces, cable lengths, spring stiffnesses and spring free lengths of mechanism $i$, respectively (where subscripts $r$ and $l$ denote left and right, respectively) and let $m_{i}$ and $y_{i}$ denote the mass and the altitude of the gravity center of mechanism $i$, respectively. Then:

$$
\begin{gathered}
U_{\text {springs }}=\frac{1}{2}\left(k_{r 1}\left(l_{r 1}-l_{r 10}\right)^{2}+k_{l 1}\left(l_{l 1}-l_{l 10}\right)^{2}+k_{r 2}\left(l_{r 2}-l_{r 20}\right)^{2}+k_{l 2}\left(l_{l 2}-l_{l 20}\right)^{2}\right) \\
U_{\text {gravity }}=g\left(m_{1} y_{1}+m_{2} y_{2}\right) \\
U_{\text {cables }}=f\left(f_{l i}, f_{r i}, l_{l i}, l_{r i}\right)
\end{gathered}
$$

The derivation of $U_{\text {cables }}$ as a function of the forces and the cable lengths depends on the actuation strategy. It will be detailed in the following sections.

The static equilibrium configurations are the minima of the potential energy. Thus, differentiating Eq. (15) with respect to $\theta_{1}$ and $\theta_{2}$ yields the relations between the forces in the cables and the configuration of the manipulator:

$$
\left\{\begin{array}{l}
\frac{\partial U_{\text {total }}}{\partial \theta_{1}}=0 \\
\frac{\partial U_{\text {total }}}{\partial \theta_{2}}=0
\end{array}\right.
$$

The Hessian matrix of the potential energy can be further calculated to analyze the stability of the equilibrium configurations and to define the stiffness matrix $\mathbf{K}_{\theta}$ of the manipulator [32]:

$$
\mathbf{K}_{\theta}=\left(\begin{array}{cc}
K_{\theta_{1}} & K_{\theta_{1} \theta_{2}} \\
K_{\theta_{1} \theta_{2}} & K_{\theta_{2}}
\end{array}\right)=\left(\begin{array}{ll}
\frac{\partial^{2} U_{\text {total }}}{\partial \theta_{1}^{2}} & \frac{\partial^{2} U_{\text {total }}}{\partial \theta_{1} \partial \theta_{2}} \\
\frac{\partial^{2} U_{\text {total }}}{\partial \theta_{1} \partial \theta_{2}} & \frac{\partial^{2} U_{\text {total }}}{\partial \theta_{2}^{2}}
\end{array}\right)
$$




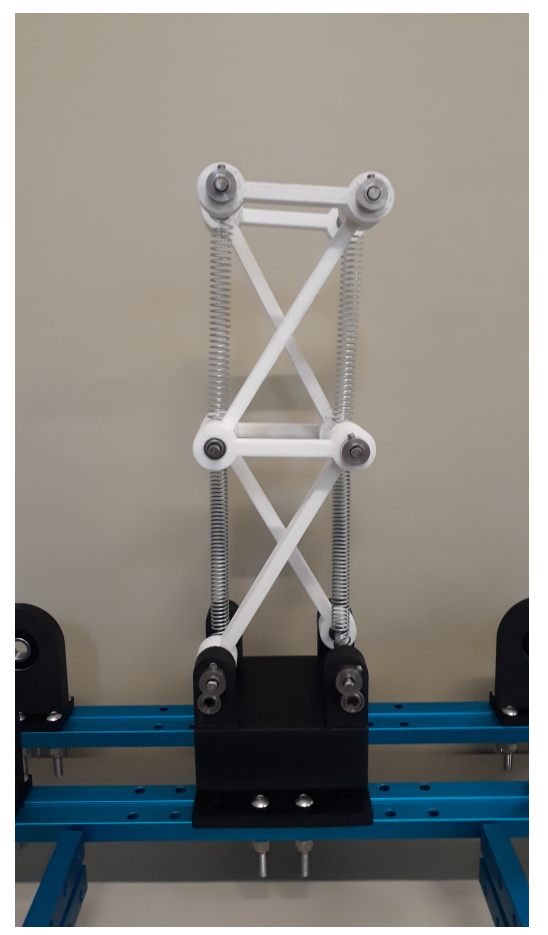

Fig. 10: Prototype in its rest configuration

The Cartesian stiffness matrix $\mathbf{K}_{\mathbf{c}}$ of the mechanism is then obtained with the Jacobian matrix $\mathbf{J}$ as:

$$
\mathbf{K}_{c}=\mathbf{J}^{-\top} \mathbf{K}_{\theta} \mathbf{J}^{-1}
$$

Controlling the joint or Cartesian stiffness can be of interest in some situations, especially for robots that may interact with obstacles and humans. Consider a task where the robot has to move among obstacles including human workers to a working point where a drilling operation must be carried out. A low joint stiffness during motion is desirable to reduce the impact of collisions, while at the goal point a high Cartesian stiffness in the drilling direction is necessary.

\subsection{Prototype data}

A first prototype is under construction for future experimental studies. It is shown here without its actuation units to provide some practical information, see Fig. 10. The assembly was arranged in space to avoid self collisions. The ratio $b / L$ was mainly decided by considerations regarding the springs extension. Since the minimal and maximal side lengths of each mechanism are $L-b$ and $L+b$, respectively, $b$ should not be too large to limit the ratio between the minimal and maximal spring lengths. A ratio $b / L=1 / 2$ allows a large choice of springs. Moreover, a compact model that can be easily handled is desired. Accordingly, the bar dimensions were fixed to $L=0.1 \mathrm{~m}$ and $b=0.05 \mathrm{~m}$. The workspace along with the number of inverse kinematic solutions in each region are shown in Fig. 11. Note that with these dimensions, the manipulator is cuspidal, but the region where nonsingular posture changing motions are feasible is restricted in the small swallowtail region on top of the workspace. The spring stiffnesses are $k_{l 1}=k_{r 1}=150 \mathrm{~N} / \mathrm{m}$ and $k_{l 2}=k_{r 2}=90 \mathrm{~N} / \mathrm{m}$ and their zero-lengths are $l_{l 10}=l_{r 10}=0.047 \mathrm{~m}$ and $l_{l 20}=l_{r 20}=0.037 \mathrm{~m}$. The chosen zero-length values insure that the springs are always in tension. The bars were 3D-printed in ABS with a rectangular section of dimension $0.01 \mathrm{~m} \times 0.005 \mathrm{~m}$. Their masses are $0.009 \mathrm{~kg}$ for the crossed bars and $0.006 \mathrm{~kg}$ for the upper and base bars. All the aforementioned data but the spring stiffnesses shall be used for the calculation of the wrench feasible workspaces in the next sections.

\subsection{Actuation strategy with two forces}

The first actuation strategy studied uses two symmetric cables routed along each side of the mechanisms, as shown in Fig.12. The work produced by the cables can be written as follows:

$$
U_{\text {cables }}=f_{l}\left(l_{l 1}+l_{l 2}\right)+f_{r}\left(l_{r 1}+l_{r 2}\right)
$$




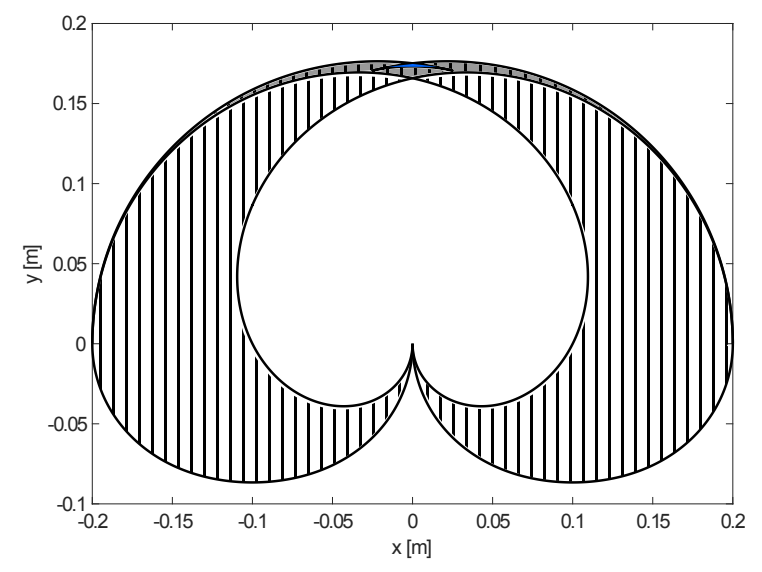

Fig. 11: Workspace of the manipulator with the prototype dimensions. The nonempty areas are filled with vertical lines. The number of solutions is defined by the filling color: none $=1$, grey $=2$ and blue $=4$

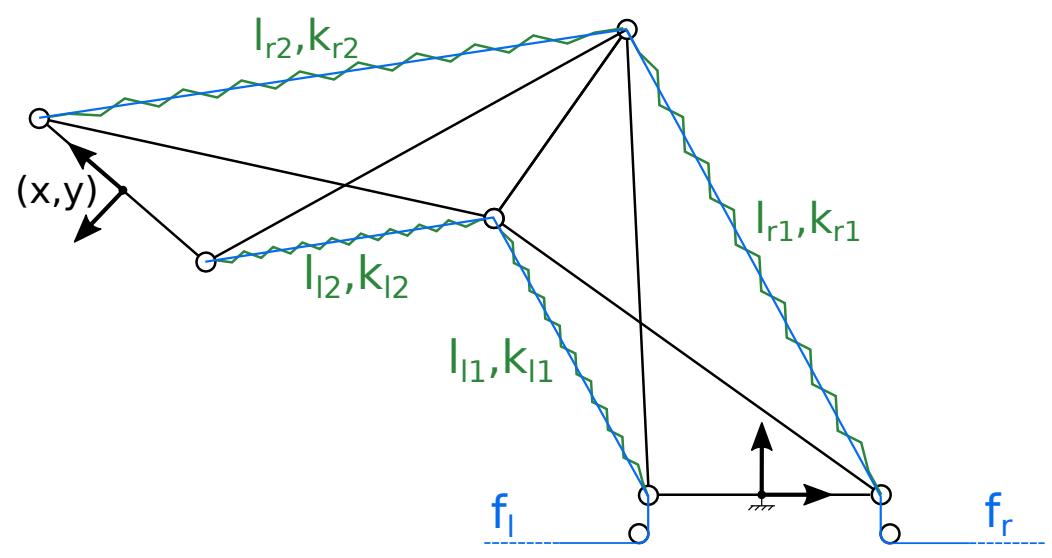

Fig. 12: First actuation strategy with two cables

where $f_{l}$ and $f_{r}$ are the cable forces applied on the left and right cable, respectively. Eq. (17) can thus be put in the following form:

$$
\left\{\begin{array}{l}
-f_{l} \frac{\partial l_{l 1}}{\partial \theta_{1}}-f_{r} \frac{\partial l_{r 1}}{\partial \theta_{1}}=k_{l 1} \frac{\partial l_{l 1}}{\partial \theta_{1}}\left(l_{l 1}-l_{l 10}\right)+k_{r 1} \frac{\partial l_{r 1}}{\partial \theta_{1}}\left(l_{r 1}-l_{r 10}\right)+g\left(m_{1} \frac{\partial y_{1}}{\partial \theta_{1}}+m_{2} \frac{\partial y_{2}}{\partial \theta_{1}}\right) \\
-f_{l} \frac{\partial l_{l 2}}{\partial \theta_{2}}-f_{r} \frac{\partial l_{r 2}}{\partial \theta_{2}}=k_{l 2} \frac{\partial l_{l 2}}{\partial \theta_{2}}\left(l_{l 2}-l_{l 20}\right)+k_{r 2} \frac{\partial l_{r 2}}{\partial \theta_{2}}\left(l_{r 2}-l_{r 20}\right)+g\left(m_{2} \frac{\partial y_{2}}{\partial \theta_{2}}\right)
\end{array}\right.
$$

This system is linear in $f_{l}$ and $f_{r}$. Accordingly, the inverse static model can be solved easily and there is one unique solution $\left(f_{l}, f_{r}\right)$ for a prescribed $\left(\theta_{1}, \theta_{2}\right)$.

The direct static problem, also known as form finding problem in tensegrity structures [3], requires solving $\left(\theta_{1}, \theta_{2}\right)$ for a prescribed $\left(f_{l}, f_{r}\right)$, which is much more difficult to handle algebraically. Indeed, when deriving the expressions of all derivative terms in system (21), complex expressions appear involving square roots of trigonometric expressions both in numerators and denominators. First numerical experiments have revealed the existence of up to seven equilibrium solutions in the ranges $-\pi / 2 \leq \theta_{1} \leq \pi / 2,-\pi / 2 \leq \theta_{2} \leq \pi / 2$, of which one or two only are stable. The complete algebraic treatment of the direct static problem turns out to be very challenging and is left to future work.

In order to find the set of points $(x, y)$ that the manipulator can reach with this actuation strategy, the joint space $\left(\theta_{1}, \theta_{2}\right)$ is discretized and the corresponding points $(x, y)$ and forces $\left(f_{l}, f_{r}\right)$ are computed. Since the cables can only pull, the forces must be always positive. A minimal positive value is assumed to ensure that the cables are always in tension. Moreover they are bounded by some maximal values depending on the actuators: $\left(f_{\min }, f_{\min }\right)<\left(f_{l}, f_{r}\right)<\left(f_{\max }, f_{\max }\right)$. The set of points $(x, y)$ that satisfy these constraints and in which the manipulator can balance a given set of external wrenches is called the wrench feasible workspace (WFW) [33]. Here, the only external wrenches considered are the gravity effects. Figure 13 shows the WFW of the manipulator for the prototype data. The WFW is shown in blue. The grey areas were added to show 

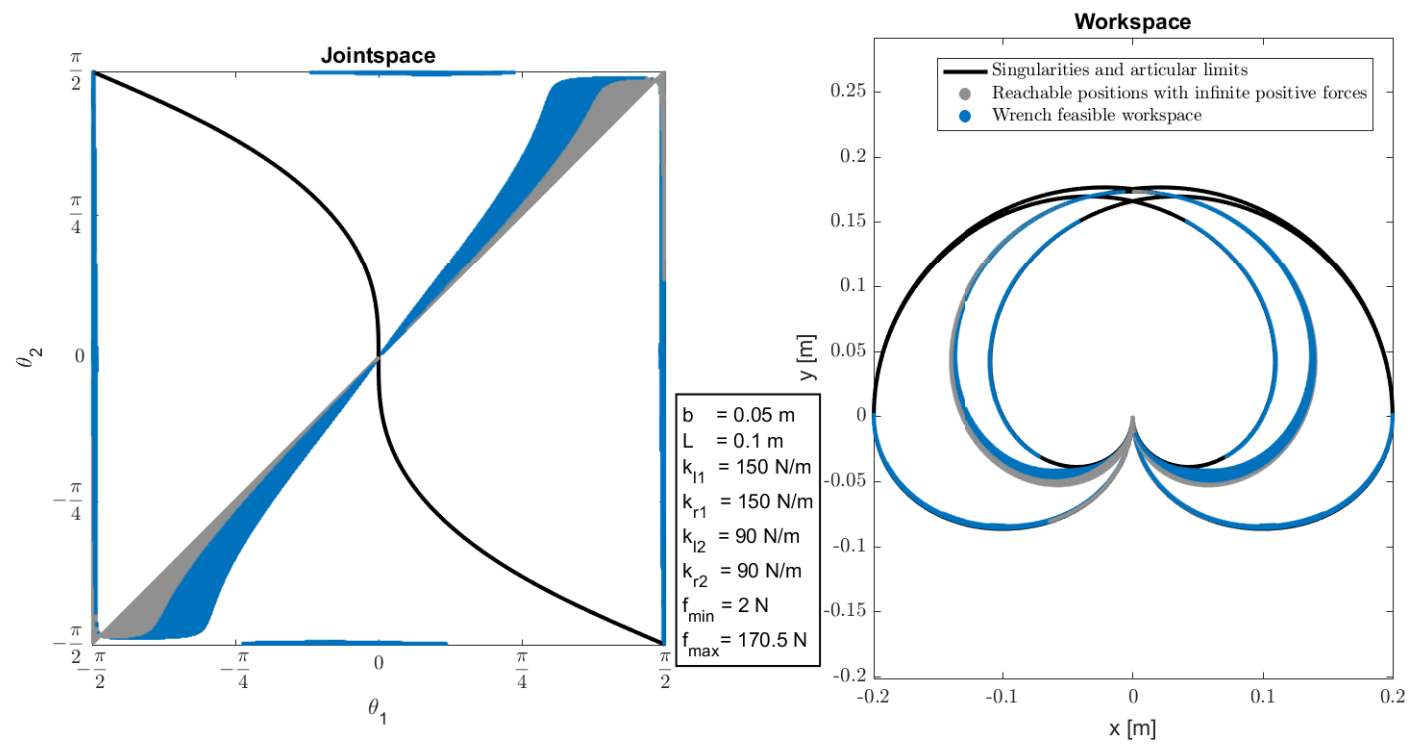

Fig. 13: The WFW in the joint (left) and Cartesian (right) space when the two mechanisms have different springs
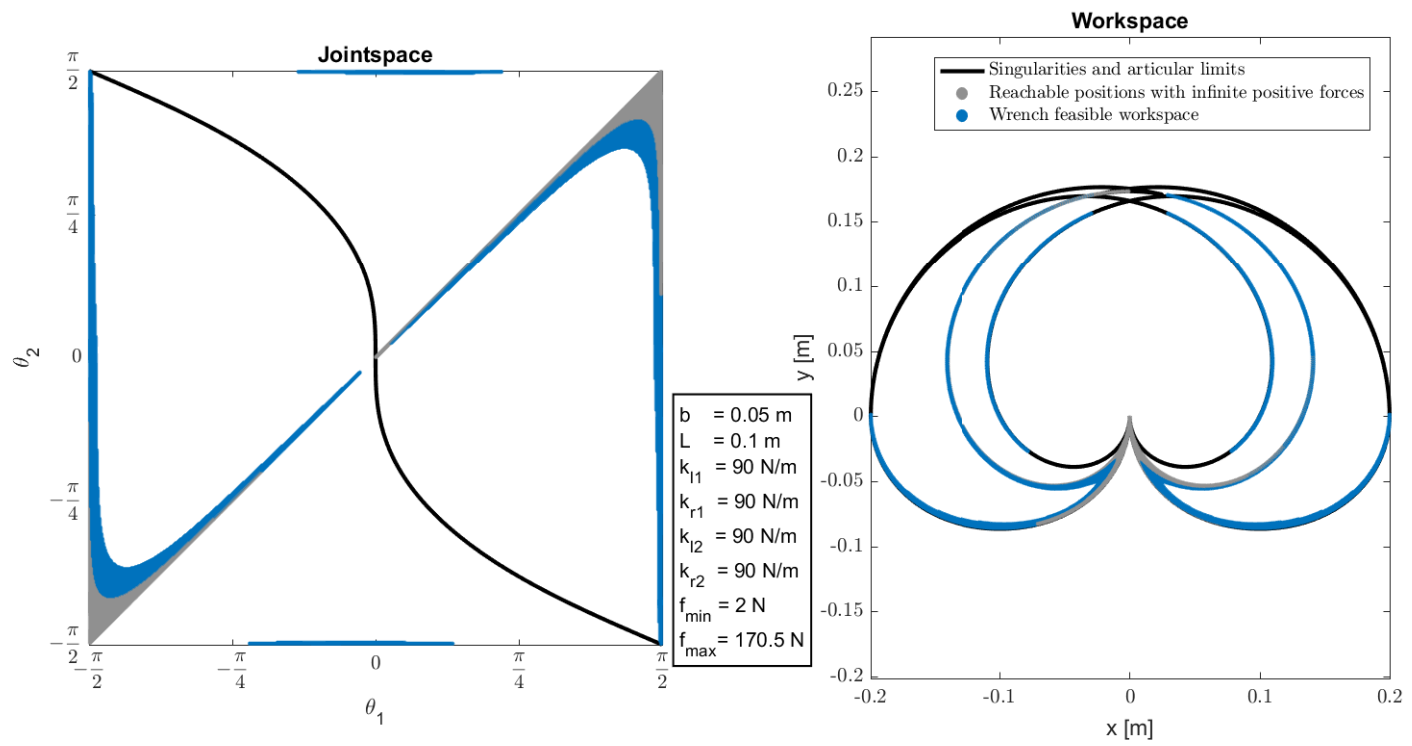

Fig. 14: The WFW in the joint (left) and Cartesian (right) space when the two mechanisms have identical springs

the solutions satisfying only the constraint $f_{l}>f_{\min }$ and $f_{r}>f_{\min }$. Clearly, the WFW is much smaller than the geometric workspace. Most points of the WFW lie in the bottom of the workspace, even when the maximum forces are not bounded. The non-symmetry of the WFW comes from the non-symmetry in the spring stiffnesses. Figure 14 shows that when all spring stiffnesses are equal, the size of the WFW is still reduced. This is because in this case the two mechanisms are identical and their behaviors differ only because of the gravity effects. Since these effects are small, most reachable configurations are located around $\theta_{1}=\theta_{2}$. Note that in the absence of gravity and with identical springs, the two mechanisms would have exactly the same behavior and the manipulator would be able to reach only configurations $\theta_{1}=\theta_{2}$, namely, it would have only one degree of freedom.

\subsection{Actuation strategy with four forces}

With the above actuation strategy, the WFW is very small. Moreover, the stiffness cannot be controlled independently of the configuration since there are only two active cables. For a control of both the configuration and the stiffness of the manipulator, four active cables are necessary. Accordingly, a second actuation strategy is studied with two more actuators 


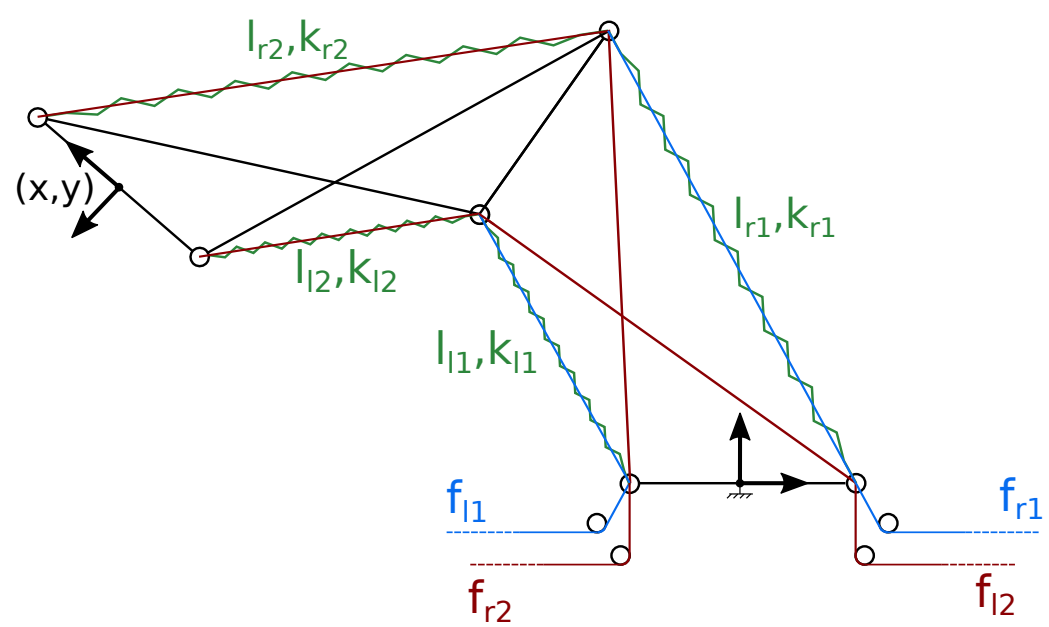

Fig. 15: Second actuation scheme with four cables. The cables driving the second mechanism go along the crossing bars of the first mechanism and then pass along the sides of the second mechanism

fixed on the base frame. These two additional motors actuate the first mechanism with two active cables routed along its sides and attached to its upper bar. The second mechanism can be driven with two cables routed along the sides of the two mechanisms like in the first actuation strategy. Another choice is a strut-routed scheme, namely, the cables pass along the crossing bars of the first mechanism. Both solutions have their own benefits and drawbacks [31]. One important drawback of the side-routed scheme is that it creates couplings in the cable lengths. Moreover, it tends to increase the stiffness of the first mechanism, since it is affected by the four forces. Accordingly, a strut-routed scheme is chosen (see Fig. 15). For this actuation strategy, The work produced by the cables is:

$$
U_{\text {cables }}=f_{l 1} l_{l 1}+f_{r 1} l_{r 1}+f_{l 2} l_{l 2}+f_{r 2} l_{r 2}
$$

As there are four forces for only two degrees of freedom, system of Eqs. (17) has twice-infinitely many solutions. The force redundancy can be solved by imposing a minimal or a maximal stiffness value of two elements in $\mathbf{K}_{\theta}$, depending on whether safe interactions are desirable or a high stiffness is required. Since the forces appear only in the diagonal elements of $\mathbf{K}_{\theta}$ (the non-diagonal terms depend only on the gravity), the two diagonal terms $K_{\theta_{1}}$ and $K_{\theta_{2}}$ can be chosen. To compute the WFW, the joint space is scanned for each configuration $\left(\theta_{1}, \theta_{2}\right)$, and the set of forces $\left(f_{l 1}, f_{l 2}, f_{r 1}, f_{r 2}\right)$ with minimal and maximal values of $K_{\theta_{1}}$ and $K_{\theta_{2}}$ is computed. Since each mechanism $i$ is actuated with two antagonistic forces $\left(f_{l i}, f_{r i}\right)$ independently from the other mechanism, the minimal stiffness $K_{\theta_{i}}$ is obtained when one of the two forces $\left(f_{l i}, f_{r i}\right)$ vanishes. An algorithm was developed to determine the two zero forces associated to $\left(\theta_{1}, \theta_{2}\right)$ and to compute the other two forces using Eq. (17). If the computed forces fall within their bounds, the corresponding stiffnesses $K_{\theta_{1}}$ and $K_{\theta_{2}}$ are then calculated using Eq. (18). A similar algorithm is used to compute the maximal stiffnesses, but this time at least two forces are set to their maximal value. Figure 16 (resp. Fig. 17) shows the wrench-feasible configurations in the joint space along with the minimal (resp. maximal) joint stiffnesses. These figures show that the joint stiffness of the $i^{\text {th }}$ mechanism mainly depends on its own $\theta_{i}$ position, but there is also a small influence of the other mechanism, which is due to the gravity effects. It can be seen here that all the configurations $\left(\theta_{1}, \theta_{2}\right)$ are inside the WFW. Some configurations near the joint limits have negative stiffness, which means that the manipulator will be in an unstable equilibrium there. This is due to the closeness to the flat singularities, that are unstable. Such configurations could be avoided by reducing the joint limits.

For each $\left(\theta_{1}, \theta_{2}\right)$, the corresponding point $(x, y)$ is calculated with Eq. (3) and the Cartesian stiffness $\mathbf{K}_{c}$ is computed using Eq. (19). The maximal and minimal values of the diagonal elements $K_{x}$ and $K_{y}$, which define the stiffness in the $x$ and $y$ direction, respectively, are computed like above (it can be shown from their algebraic expressions that $K_{x}$ and $K_{y}$ attain their maximum and minimum values for the same forces as $K_{\theta_{1}}$ and $K_{\theta_{2}}$ ). Figure 18 (resp. Fig. 19) shows the WFW along with the minimal and maximal Cartesian stiffnesses in the $x$ and $y$ directions. The Cartesian stiffness is naturally influenced by the manipulator singularities as well as by the mechanism singularities. They even become infinite in the direction defined by the two instantaneous centers of rotation and the end-effector point. To avoid infinite values, the calculation of the Cartesian stiffness was discarded around the singularities. It is worth noting that there is generally a ratio of 10 between the minimal and the maximal stiffnesses both in the joint space and in the Cartesian space, which allows tuning the stiffness in a rather large range. Note that the stiffness mapping was conducted in the $x$ and $y$ directions, but any other directions could have been considered. 

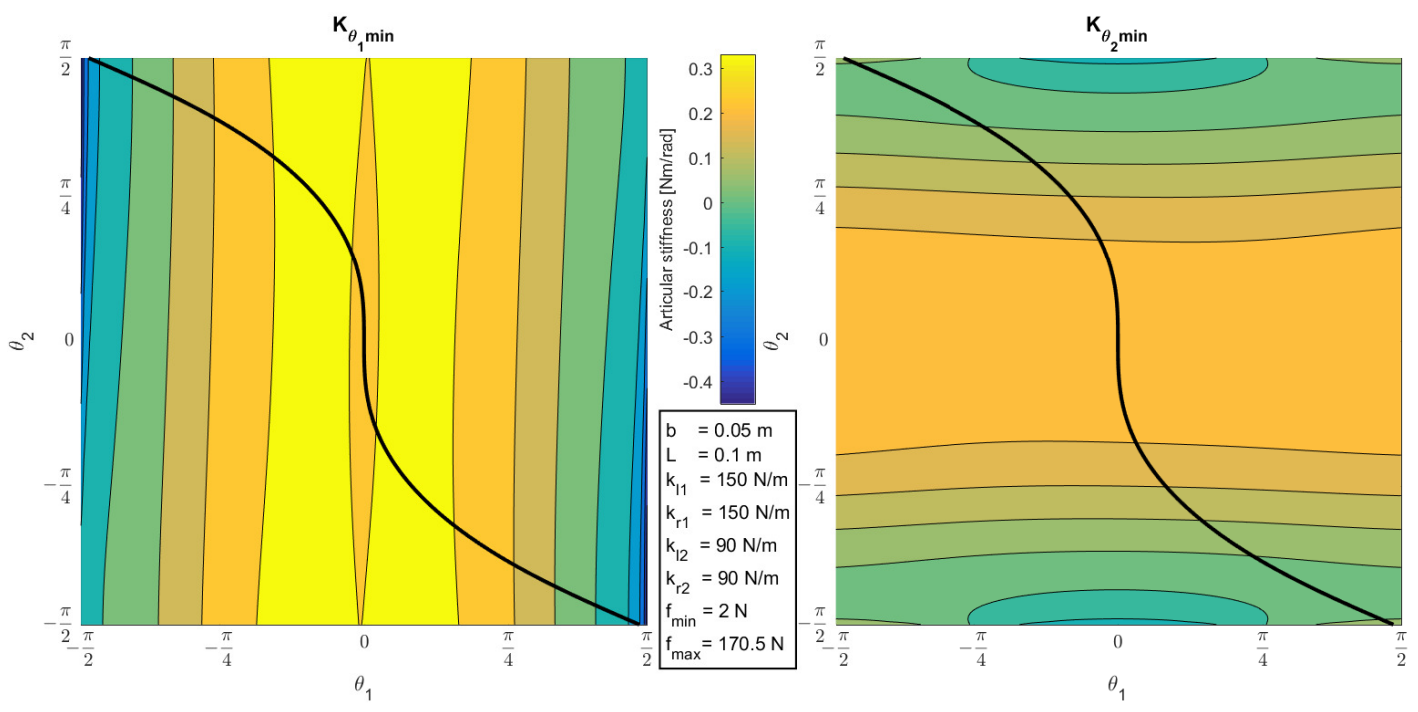

Fig. 16: Minimal joint stiffnesses
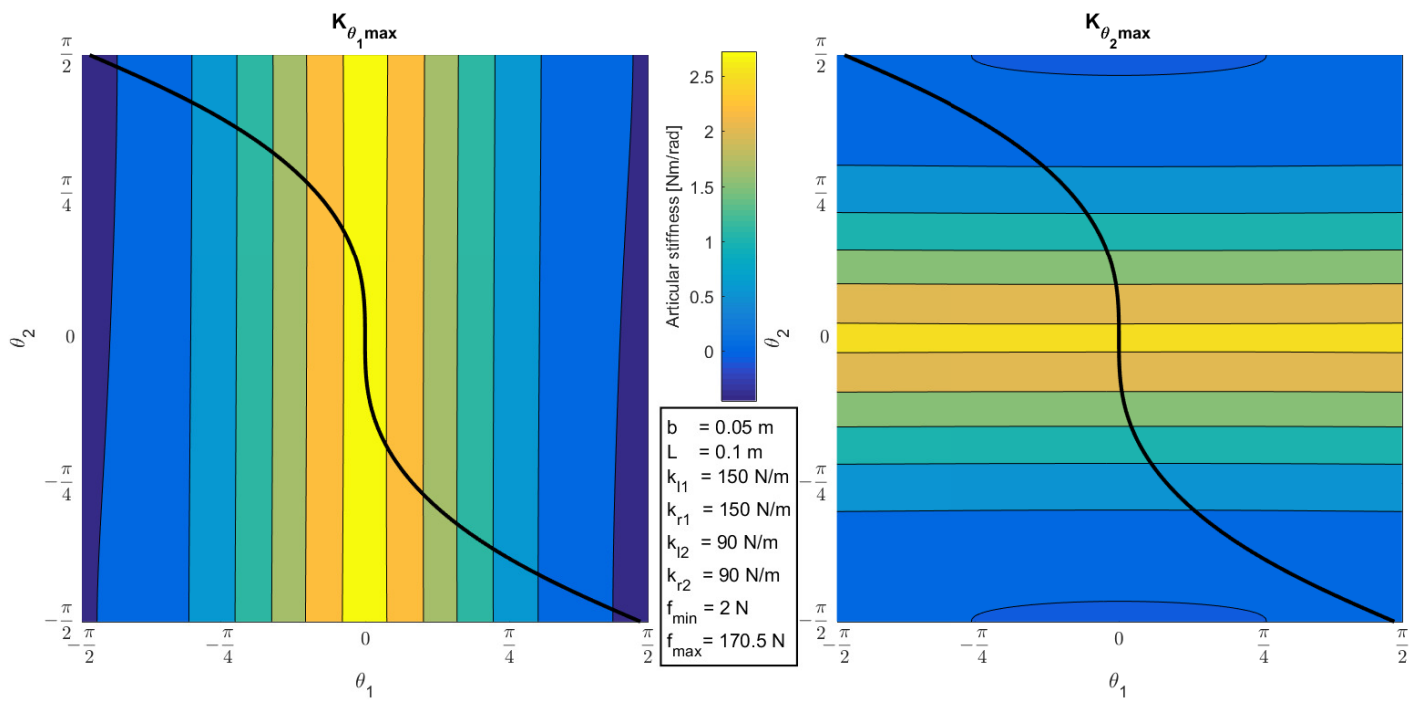

Fig. 17: Maximal joint stiffnesses

\section{Conclusion}

This paper has reported on a preliminary study of a project involving biologists that aims at producing a bird neck model as a bio-inspired manipulator design with enhanced performances. Accordingly, a 2-degree-of-freedom tensegrity manipulator made of two X-mechanisms in series has been studied. Although simple at a first glance, this manipulator turned out to have some challenging kinematics issues, such as an inverse kinematics that requires an adequate solving methodology and a cuspidal behavior. On the other hand, it lends itself well to remote actuation schemes with cables and springs. The first part was dedicated to a pure kinematic analysis, where a methodology to solve the inverse kinematics was proposed, the singularities were determined and the workspace with and without joint limits was analyzed in details. The manipulator was shown to have two or four solutions depending on its geometric parameter values. When it has four solutions, the manipulator was shown to be cuspidal, a result that was never encountered before in a planar 2-dof manipulator, to the best of the authors' knowledge. A cuspidality condition based on the geometric parameters was established. In the second part, two actuation schemes with remote motors, lateral springs and cables have been proposed. The first scheme uses only two motors, which is enough from a pure kinematic point of view. However, this scheme turned out to produce a limited wrench feasible workspace (WFW). The second actuation strategy uses four remote motors, thus introducing actuation redundancy. In addition to producing a much larger WFW, this redundant actuation strategy allows tuning the stiffness in the workspace. This feature is of high interest for tasks requiring both safe interactions and high forces transmission. Only static analyses have been reported in this paper. The dynamic behavior is under study and first simulation results with a dynamic control 

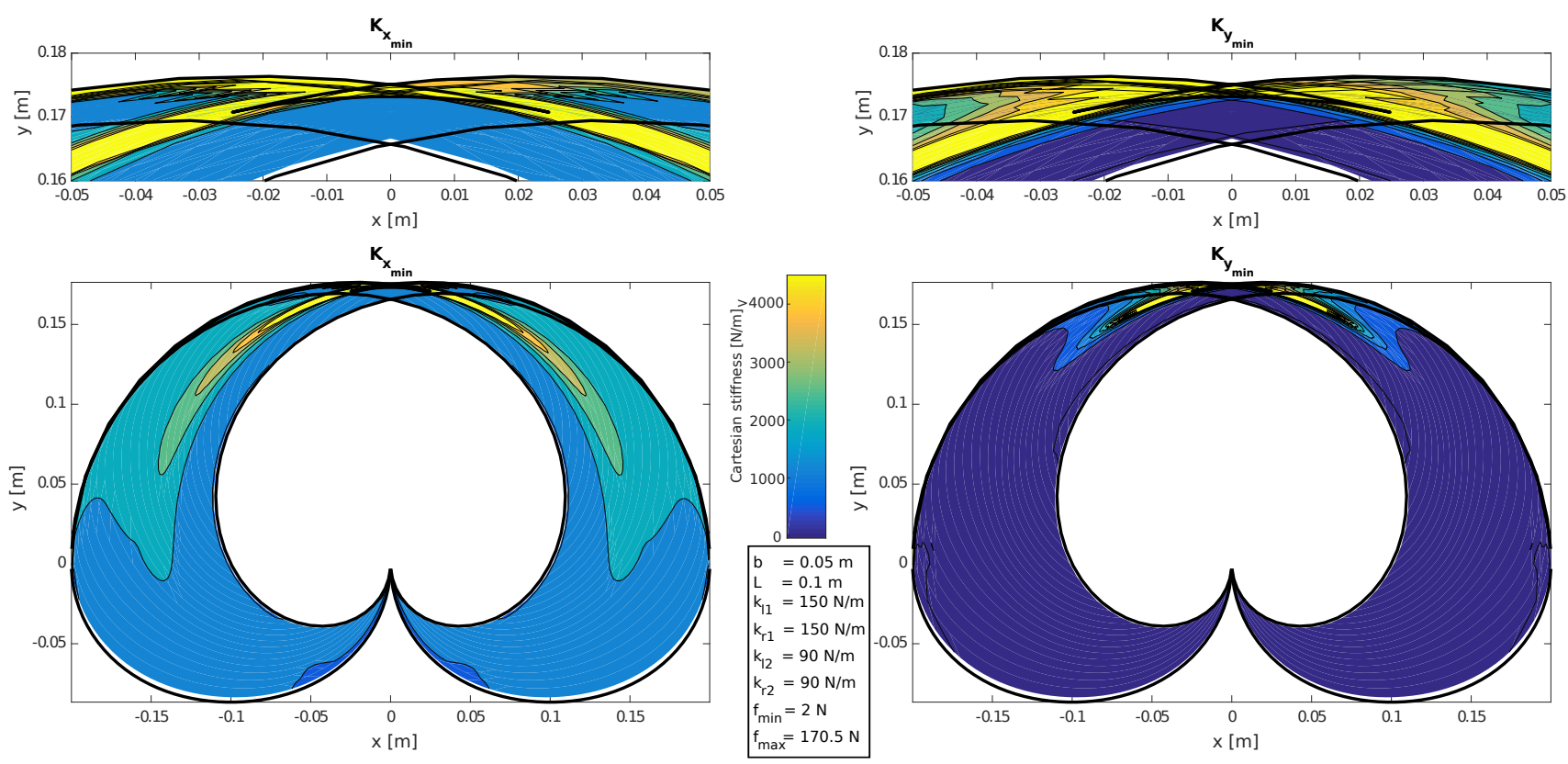

Fig. 18: Wrench feasible workspace with the minimal Cartesian stiffness mapping
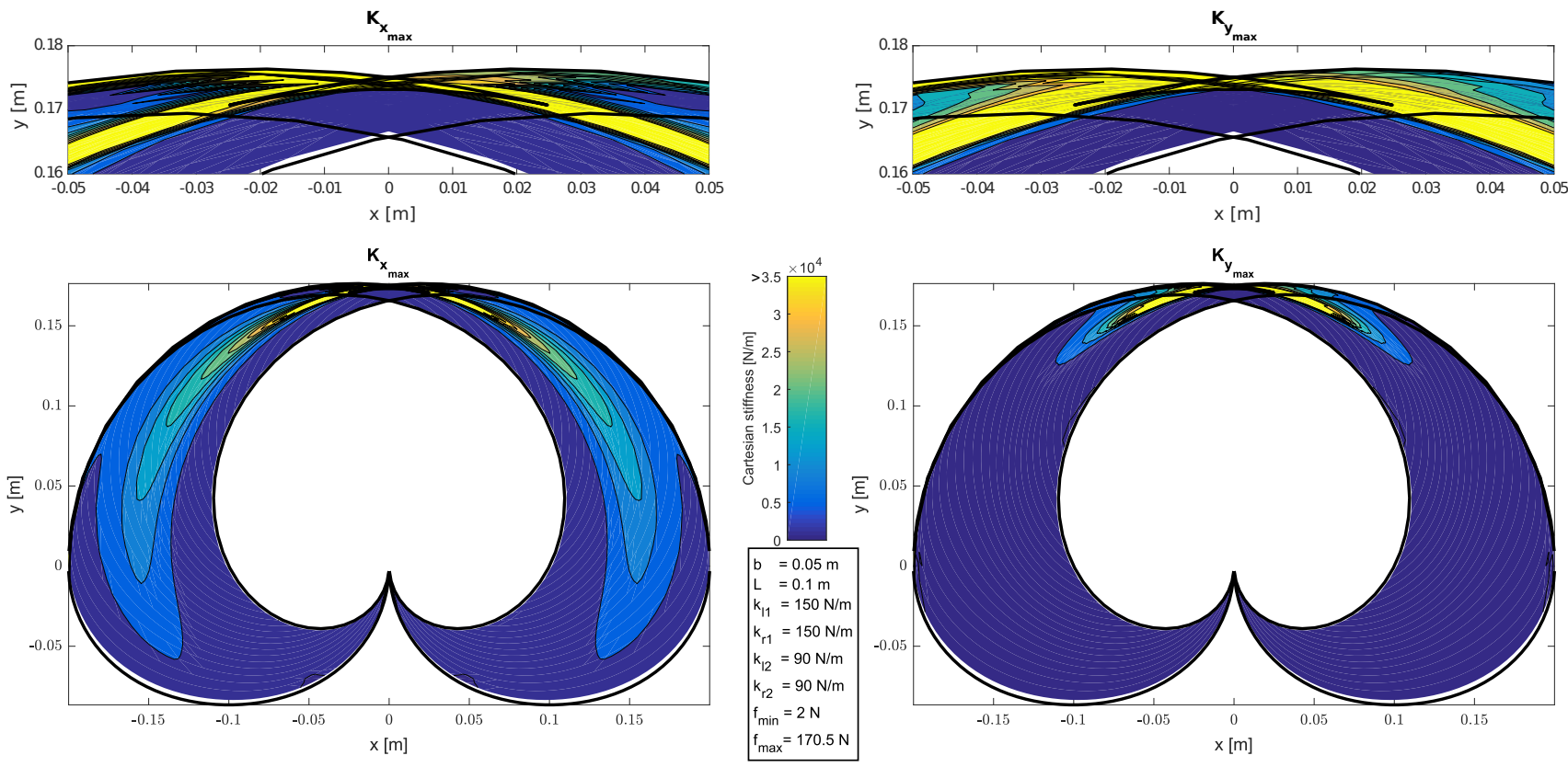

Fig. 19: Wrench feasible workspace with the maximal Cartesian stiffness mapping

law reveal satisfactory responses [34]. Experiments are now being conducted on a prototype and shall be presented in a future paper. First results show that friction, which was neglected in this study, has signification effects. It can be taken into account by adding a static force as an offset in the active forces. In future work, the planar model will be completed with more mechanisms in series. Kinematic redundancy will allow avoiding obstacles, and even shaping around them, where contacts with obstacles will be modelled with external forces. Finally, a 3D model will be proposed, which will account for the additional degree of freedom existing between two subsequent vertebrae in the real bird neck.

\section{Acknowledgements} 0025).

This work was conducted with the support of the French National Research Agency (AVINECK Project ANR-16-CE33- 


\author{
Nomenclature \\ $L$ Diagonal bar length \\ $b$ Bottom and top bar length \\ $\theta_{i} \quad$ Median bar orientation of mechanism $i$ \\ $l_{i} \quad$ Median bar length of mechanism $i$ \\ J Jacobian matrix of the manipulator \\ $k_{r i} \quad$ Right spring stiffness of mechanism $i$ \\ $k_{l i} \quad$ Left spring stiffness of mechanism $i$ \\ $l_{r i} \quad$ Right spring length of mechanism $i$ \\ $l_{l i} \quad$ Left spring length of mechanism $i$ \\ $l_{r i 0} \quad$ Right spring zero free length of mechanism $i$ \\ $l_{l i 0} \quad$ Left spring zero free length of mechanism $i$ \\ $f_{r i} \quad$ Force along right spring of mechanism $i$ \\ $f_{l i} \quad$ Force along left spring of mechanism $i$ \\ $g$ Gravity acceleration \\ $m_{i} \quad$ Mass of mechanism $i$ \\ $y_{i} \quad$ Height of CoM of mechanism $i$ \\ $U_{\text {total }}$ Total potential energy of manipulator \\ $\mathbf{K}_{\theta} \quad$ Hessian matrix of manipulator \\ $\mathbf{K}_{\mathbf{c}}$ Cartesian stiffness matrix of manipulator
}

\title{
References
}

[1] Buckminster, F. R., 1962. Tensile-integrity structures, Nov. 13. US Patent 3,063,521.

[2] Motro, R., 1992. "Tensegrity systems: the state of the art". International journal of space structures, 7(2), June, pp. $75-83$.

[3] Skelton, R. E., and de Oliveira, M. C., 2009. Tensegrity systems, Vol. 1. Springer, Boston, MA.

[4] Levin, S. M., 2002. "The tensegrity-truss as a model for spine mechanics: biotensegrity". Journal of mechanics in medicine and biology, 2(03n04), pp. 375-388.

[5] Arsenault, M., and Gosselin, C. M., 2006. "Kinematic, static and dynamic analysis of a planar 2-dof tensegrity mechanism". Mechanism and Machine Theory, 41(9), September, pp. 1072-1089.

[6] Crane, C. D., Bayat, J., Vikas, V., and Roberts, R., 2008. "Kinematic analysis of a planar tensegrity mechanism with pre-stressed springs". In Advances in Robot Kinematics: analysis and design, L. J. and W. P., eds. pp. 419-427.

[7] Wenger, P., and Chablat, D., 2017. "Kinetostatic analysis and solution classification of a planar tensegrity mechanism". In Proceedings of the 7th International Workshop on Computational Kinematics, May 22-24, 2017, Poitiers, France, L. M. Zeghloul S., Romdhane L., ed., Springer, pp. 422-431.

[8] Rieffel, J., and Mouret, J.-B., 2018. "Adaptive and resilient soft tensegrity robots". Soft robotics, 5(3), june, pp. 318329.

[9] Böhm, V., Kaufhold, T., Schale, F., and Zimmermann, K., 2016. "Spherical mobile robot based on a tensegrity structure with curved compressed members". In 2016 IEEE International Conference on Advanced Intelligent Mechatronics (AIM), July 12-15, 2016, Banff, AB, Canada, IEEE, pp. 1509-1514.

[10] Vespignani, M., Friesen, J. M., SunSpiral, V., and Bruce, J., 2018. "Design of superball v2, a compliant tensegrity robot for absorbing large impacts". In 2018 IEEE/RSJ International Conference on Intelligent Robots and Systems (IROS), October 1-5, 2018, Madrid, Spain, IEEE, pp. 2865-2871.

[11] Bakker, D. L., Matsuura, D., Takeda, Y., and Herder, J. L., 2015. "Design of an environmentally interactive continuum manipulator". In Proceedings of 14th IFToMM World Congress in Mechanism and Machine Science, October 25-30, 2015, Taipei, Taiwan,, pp. $327-336$.

[12] Snelson, K. D., 1965. Continuous tension, discontinuous compression structures, Feb. 16. US Patent 3,169,611.

[13] Zweers, G., Bout, R., and Heidweiller, J., 1994. "Motor organization of the avian head-neck system". In Perception and motor control in birds : An ecological approach, M. N. O. Davies and P. R. Green, eds. Springer, Dordrecht, Germany, pp. 201-221.

[14] Boehler, Q., Charpentier, I., Vedrines, M. S., and Renaud, P., 2015. "Definition and computation of tensegrity mechanism workspace". Journal of Mechanisms and Robotics, 7(4), Nov, p. 044502.

[15] Van Riesen, A., Furet, M., Chevallereau, C., and Wenger, P., 2018. "Dynamic analysis and control of an antagonistically actuated tensegrity mechanism". In Proocedings of 22nd CISM IFToMM Symposium on Robot Design, Dynamics and Control (ROMANSY), June 25-28, 2018, Rennes, France, Springer, pp. 481-490. 
[16] Aldrich, J., and Skelton, R., 2005. "Time-energy optimal control of hyper-actuated mechanical systems with geometric path constraints". In Proceedings of the 44th IEEE Conference on Decision and Control, December 15, 2005, Seville, Spain, IEEE, pp. 8246-8253.

[17] Arsenault, M., and Gosselin, C. M., 2006. "Kinematic and static analysis of a planar modular 2-dof tensegrity mechanism". In Proceedings of 2006 IEEE International Conference on Robotics and Automation (ICRA), May 15-19, 2006, Orlando, FL, USA, IEEE, pp. 4193-4198.

[18] Chen, S., and Arsenault, M., 2012. "Analytical computation of the actuator and cartesian workspace boundaries for a planar 2-degree-of-freedom translational tensegrity mechanism". Journal of Mechanisms and Robotics, 4(1), February, p. 011010.

[19] Furet, M., Van Riesen, A., Chevallereau, C., and Wenger, P., 2018. "Optimal design of tensegrity mechanisms used in a bird neck model". In Proceedings of the 7th European Conference on Mechanism Science (EuCoMeS), September 4 - 6, 2018, Aachen, Germany, Springer, pp. 365-375.

[20] Furet, M., Lettl, M., and Wenger, P., 2018. "Kinematic analysis of planar tensegrity 2-x manipulators". In Proceedings of the 16th International Symposium on Advances in Robot Kinematics (ARK), July 1-5, Bologna, Italia, Springer, pp. 153-160.

[21] Furet, M., and Wenger, P., 2018. "Workspace and cuspidality analysis of a 2-x planar manipulator". In Proceedings of the 4th IFToMM Symposium on Mechanism Design for Robotics (MEDER), September 11 - 13, 2018, Udine, Italy, Springer, pp. 110-117.

[22] Jha, R., Chablat, D., Baron, L., Rouillier, F., and Moroz, G., 2018. "Workspace, joint space and singularities of a family of delta-like robot". Mechanism and Machine Theory, 127, September, pp. 73-95.

[23] Manubens, M., Moroz, G., Chablat, D., Wenger, P., and Rouillier, F., 2012. "Cusp points in the parameter space of degenerate 3-rpr planar parallel manipulators". Journal of mechanisms and robotics, 4(4), p. 041003.

[24] Moroz, G., Rouiller, F., Chablat, D., and Wenger, P., 2010. "On the determination of cusp points of 3-rpr parallel manipulators". Mechanism and Machine Theory, 45(11), pp. 1555-1567.

[25] Borrel, P., and Liégeois, A., 1986. "A study of multiple manipulator inverse kinematic solutions with applications to trajectory planning and workspace determination". In Proceedings of 1986 IEEE International Conference on Robotics and Automation (ICRA), April 7-10, 1986, San Francisco, CA, USA, Vol. 3, IEEE, pp. 1180-1185.

[26] El Omri, J., and Wenger, P., 1995. "How to recognize simply a non-singular posture changing 3-dof manipulator". In Proceedings of 7th International Conference on Advanced Robotics (ICAR), September 20-22, 1995, Sant Feliu de Guixois, Spain, pp. 215-222.

[27] Wenger, P., 2007. "Cuspidal and noncuspidal robot manipulators". Robotica, 25(6), November, pp. 677-689.

[28] Derivation of a polynomial equation for the boundaries of 2-x manipulators. Tech. rep., Laboratoire des sciences du numérique de Nantes (LS2N).

[29] Thomas, F., and Wenger, P., 2011. "On the topological characterization of robot singularity loci. a catastrophe-theoretic approach". In Proceedings of 2011 IEEE International Conference on Robotics and Automation (ICRA), May 9-13 2011, Shanghai, China, IEEE, pp. 3940-3945.

[30] Wenger, P., Chablat, D., and Baili, M., 2005. "A dh-parameter based condition for 3r orthogonal manipulators to have four distinct inverse kinematic solutions". Journal of Mechanical Design, 127(1), March, pp. 150-155.

[31] Moored, K., Kemp, T., Houle, N., and Bart-Smith, H., 2011. "Analytical predictions, optimization, and design of a tensegrity-based artificial pectoral fin". International Journal of Solids and Structures, 48(22-23), november, pp. 31423159.

[32] Quennouelle, C., and Gosselin, C. "Stiffness matrix of compliant parallel mechanisms". In Advances in Robot Kinematics: Analysis and Design, L. J. and W. P., eds. pp. 331-341.

[33] Ebert-Uphoff, I., and Voglewede, P. A., 2004. "On the connections between cable-driven robots, parallel manipulators and grasping". In Proceedings of 2004 IEEE International Conference on Robotics and Automation (ICRA), 26 April-1 May, 2004, New Orleans, LA, USA, Vol. 5, IEEE, pp. 4521-4526.

[34] Fasquelle, B., Furet, M., Chevallereau, C., and Wenger, P., 2019. "Dynamic modeling and control of a tensegrity manipulator mimicking a bird neck". In Proceedings of 15th IFToMM World Congress on Mechanism and Machine Science, June 30-July 4, 2019, Krakow, Poland, Springer, pp. 2087-2097. 


\section{List of Figures}

1 An X-mechanism with two lateral springs $(a)$ and a series assembly mimicking a bird neck $(b) \ldots \ldots$

Manipulator description . . . . . . . . . . . . . . . . . . . . . . 3

The four inverse solutions at $x=0.03$ and $y=1.6(L=1$ and $b=2 / 3) \ldots \ldots$. . . . . . . . . . .

Singularity curves in the joint space and aspects when $L=1$ and $b=2 / 3$. . . . . . . . . . . . . . . . . . 6

5 Workspace boundaries when $L=1$ and $b=2 / 5(a), b=2 / 3(b), b=9 / 10(c)$. Four-solutions regions are filled in gray . . . . . . . . . . . . . . . . . . . . . . . . . . . . . .

6 Workspace boundaries for the two degenerate designs $b=L=1(a)$ and $b=0, L=1(b)$. Four-solutions regions are filled in gray . . . . . . . . . . . . . . . . . . . . . . . 7

$7 \quad \mathrm{X}$-mechanism in configuration up (a) and down $(\mathrm{b}) \ldots \ldots \ldots$

8 workspace regions with different numbers and types of solutions $\left(L=1, b=2 / 3 . \overline{M_{i}}\right.$ (resp. $\left.M_{i}\right)$ means that mechanism $i$ is in configuration down (resp. up) . . . . . . . . . . . . . . . . . . . . . . .

9 workspace boundaries when $-\pi / 2<\theta_{1}, \theta_{2}<\pi / 2$, and for $L=1, b=2 / 5$ (left), $b=2 / 3$ (center), $b=9 / 10$ (right). the nonempty part is depicted with vertical lines. The number of solutions is defined by the filling color: none $=1$, grey $=2$, red $=3$ and blue $=4 \ldots \ldots \ldots$

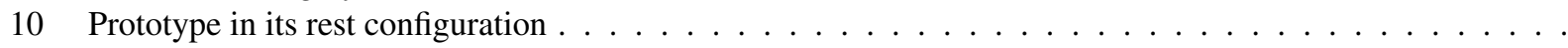

11 Workspace of the manipulator with the prototype dimensions. The nonempty areas are filled with vertical lines. The number of solutions is defined by the filling color: none=1, grey=2 and blue=4 . . . . . . . . . 12

12 First actuation strategy with two cables . . . . . . . . . . . . . . . . . . . 12

13 The WFW in the joint (left) and Cartesian (right) space when the two mechanisms have different springs . . 13

14 The WFW in the joint (left) and Cartesian (right) space when the two mechanisms have identical springs . . 13

15 Second actuation scheme with four cables. The cables driving the second mechanism go along the crossing bars of the first mechanism and then pass along the sides of the second mechanism . . . . . . . . . . 14

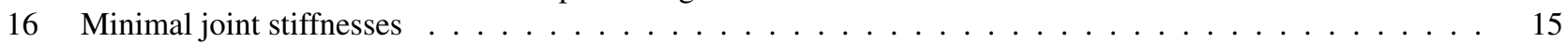

17 Maximal joint stiffnesses . . . . . . . . . . . . . . . . . . . . . . . 15

18 Wrench feasible workspace with the minimal Cartesian stiffness mapping . . . . . . . . . . . . . . 16

19 Wrench feasible workspace with the maximal Cartesian stiffness mapping . . . . . . . . . . . . . 16 